S. Szabo

U.S. DEPARTMENT OF COMMERCE National Bureau of Standards Center for Manufacturing Engineering Robot Systems Division

Gaithersburg, Maryland 20899

September 1987

Sponsored by:

United States Army

Human Engineering Laboratory

Aberdeen Proving Ground, Maryland 21005-5001 



\section{THE NATIONAL BUREAU OF STANDARDS PROGRAMMERS GUIDE FOR THE FIELD MATERIEL-HANDLING ROBOT (FMR)}

S. Szabo

U.S. DEPARTMENT OF COMMERCE

National Bureau of Standards

Center for Manufacturing Engineering

Robot Systems Division

Gaithersburg, Maryland 20899

September 1987

Sponsored by:

United States Army

Human Engineering Laboratory

Aberdeen Proving Ground, Maryland 21005-5001

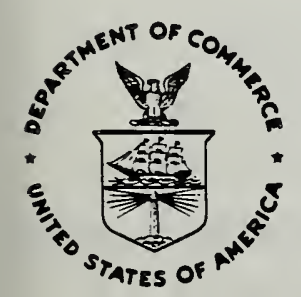

U.S. DEPARTMENT OF COMMERCE, Clarence J. Brown, Acting Secretary NATIONAL BUREAU OF STANDARDS, Ernest Ambler, Director 

1. Introduction

1.1. Documentation Conventions. . . . . . . . . . . . . . 1

2. Sensor Modelling___ $\underline{2}$

2.1. Sonar Modelling. . . . . . . . . . . . . . . . . 2

2.1.1. Model Data for the Sonar. . . . . . . . . . . . . 2

2.1.2. Compiling the Sonar Model. . . . . . . . . . . . 2

2.1.3. Interpreting the Sonar Model. . . . . . . . . . . . 3

2.1.4. Calibrating the Sonar Model. . . . . . . . . . . . 3

2.2. Proximity Detector Modelling. . . . . . . . . . . . . . 3

2.2.1. Model Data for the Proximity Dectectors. . . . . . . . . 5

2.2.2. Compiling the Proximity Dectector Model. . . . . . . . 5

2.2.3. Interpreting the Proximity Dectector Model. . . . . . . . 5

2.2.4. Calibrating the Proximity Dectector Model. . . . . . . . 5

3. Path Point Command for the FMR__

3.1. Range Path Point Command. . . . . . . . . . . . . . 9

3.2. Edge Path Point Command. . . . . . . . . . . . . . 9

3.3. Equate Path Point Command. . . . . . . . . . . . . . 9

3.4. Scan Path Point Command. . . . . . . . . . . . . 10.

3.5. Align-grip Path Point Command. . . . . . . . . . . . . 11

3.6. Approach-pallet Path Point Command. . . . . . . . . . . 12

3.7. Pickup-pallet Path Point Command. . . . . . . . . . . . 12

3.8. Goto-until-sw Path Point Command. . . . . . . . . . . . 13

3.9. Return-pose Path Point Command. . . . . . . . . . . . 13

3.10. Delay Path Point Command. . . . . . . . . . . . . . 13

4. Programming the FMR Using RSL___ 14

4.1. Transfer of a Randomly Located $155 \mathrm{~mm}$ Pallet. . . . . . . . . 14

4.1.1. The RSL Plan for Random Pallet. . . . . . . . . . . 14

4.1.2. The RSL Command to Transfer a Random Pallet. . . . . . 17

4.2. Transfer of a Randomly Located Array of $155 \mathrm{~mm}$ Pallets. . . . : . .17

4.2.1. The RSL Plan to Transfer an Array of Pallets. . . . . . . 17

4.2.2. The RSL Command to Transfer an Array of Pallets. . . . . 21

5. The FMR PATH Level___ 22

5.1 PATH Commands. . . . . . . . . . . . . . . . . 22

5.2. PATH Input Command Buffer. . . . . . . . . . . . . 22

5.3. PATH Status Information. . . . . . . . . . . . . . . 22

5.4. PATH Errors. . . . . . . . . . . . . . . . . . 23 
5.5. PATH Processing. . . . . . . . . . . . . . . . . 23

5.6. PATH Preprocessing. . . . . . . . . . . . . . . . 23

5.6.1. SONAR-READ. . . . . . . . . . . . . . . . 23

5.6.1.1. SELECT-SONAR. . . . . . . . . . . . . 23

5.6.1.2. RESET-SONAR。 . . . . . . . . . . . . . 24

5.6.1.3. OUTPUT-SONAR-SELECTION. . . . . . . . . 24

5.6.1.4. OUTPUT-SONAR-MODE. . . . . . . . . . . 24

5.6.1.5. READ-SONAR-DATA. . . . . . . . . . . . 24

5.6.1.6. GET-SONAR-STATUS. . . . . . . . . . . 24

5.6.1.7. SET-SONAR-RANGE. . . . . . . . . . . . 25

5.6.1.8. GET-SONAR-DATA. . . . . . . . . . . . 25

5.6.1.9. CLEAR-SONAR-REQUEST. . . . . . . . . . 25

5.6.1.10. SONAR-OFF. . . . . . . . . . . . . . 25

5.7. PATH Decision Processing. . . . . . . . . . . . . . 25

5.7.1. SEND-HALT. . . . . . . . . . . . . . 25

5.7.2. HALT. . . . . . . . . . . . . . . . . 26

5.7.3. TRANSLATE. . . . . . . . . . . . 26

5.7.4. ROTATE. . . . . . . . . . . . . . . . . 26

5.7.5. INIT-SONAR-MODEL-ARRAY. . . . . . . . . . . 27

5.7.6. RANGE Path Point Routines. . . . . . . . . . . . 27

5.7.6.1. RANGE-PPT. . . . . . . . . . . . . . 27

5.7.6.2. RANGE-PPT-INIT. . . . . . . . . . . . . 27

5.7.6.3. RANGE-SONAR-MODEL-INIT. . . . . . . . . 27

5.7.6.4. RANGE. . . . . . . . . . . . . . . . 27

5.7.6.5. RANGE-GOAL. . . . . . . . . . . . . . 28

5.7.7. EDGE Path Point Routines. . . . . . . . . . . . 28

5.7.6.1. EDGE-PPT. . . . . . . . . . . . . . . 28

5.7.6.2. EDGE-PPT-INIT. . . . . . . . . . . . . 28

5.7.6.3. EDGE-SONAR-MODEL-INIT. . . . . . . . . 28

5.7.6.4. EDGE. . . . . . . . . . . . . . . . . 28

5.7.6.5. ?EDGE. . . . . . . . . . . . . . . . 29

5.7.8. EQUATE Path Point Routines. . . . . . . . . . . . 29

5.7.8.1. EQUATE-PPT. . . . . . . . . . . . . . 29

5.7.8.2. EQUATE-PPT-INIT. . . . . . . . . . . . 29

5.7.8.3. EQUATE-SONAR-MODEL-INIT. . . . . . . . 29 
5.7.8.4. EQUATE. . . . . . . . . . . . . . . . 30

5.7.8.5. NOT-EQUATED. . . . . . . . . . . . . 30

5.7.9. SCAN Path Point Routines. . . . . . . . . . . . 31

5.7.9.1. SCAN-SONAR. . . . . . . . . . . . . . 31

5.7.9.2. SCAN-INIT. . . . . . . . . . . . . . 31

5.7.9.3. SCAN-SONAR-MODEL-INIT. . . . . . . . . 31

5.7.9.4. SAVE-SCAN-READING. . . . . . . . . . 31

5.7.9.5. ADD-TO-SONAR-REC. . . . . . . . . . . 31

5.7.9.6. ADD-RECORD. . . . . . . . . . . . . 32

5.7.9.7. SET-PALLET-MIN-RANGE. . . . . . . . . . 32

5.7.9.8. LEFT-EDGE-TEST. . . . . . . . . . . . . 32

5.7.9.9. LEFT-EDGE-ADJUST. . . . . . . . . . . . 32

5.7.9.10. RIGHT-EDGE-TEST. . . . . . . . . . . . 32

5.7.9.11. RIGHT-EDGE-ADJUST. . . . . . . . . . . 33

5.7.9.12. RETURN-SCAN-POSE. . . . . . . . . . . 33

5.7.9.13. FIND-LEFT-CORNER-EDGE. . . . . . . . . 34

5.7.9.14. FIND-RIGHT-CORNER-EDGE. . . . . . . . 34

5.7.9.15. SET-RETURN-POSE. . . . . . . . . . . . 34

5.7.9.16. N1-N2-SET. . . . . . . . . . . . . . . 34

5.7.10. ALIGN-GRIP Path Point Routines. . . . . . . . . . 34

5.7.10.1. ALIGN-GRIP. . . . . . . . . . . . 35

5.7.10.2. GRIP-RETRIEVE. . . . . . . . . . . . . 35

5.7.10.3. TURN-ON-SONAR. . . . . . . . . . . 35

5.7.10.4. TEST-FOR-ALIGNMENT. . . . . . . . . . 35

5.7.10.5. FIND-GRIP-SIDE-STATE. . . . . . . . . . 35

5.7.10.6. TILT-PROCESSING. . . . . . . . . . . . 36

5.7.10.7. SHORT-SIDE-PROCESSING. . . . . . . . . 36

5.7.10.8. LONG-SIDE-PROCESSING. . . . . . . . . 36

5.7.10.9. SIDE-EQUATE. . . . . . . . . . . . . 37

5.7.10.10. SIDE-EQUATE-INIT. . . . . . . . . . 37

5.7.10.11. SIDE-EDGE-INIT. . . . . . . . . . . . 37

5.7.10.12. SIDE-MOVE. . . . . . . . . . . . . . 37

5.7.10.13. SIDE-MOVE-INIT. . . . . . . . . . . . 37

5.7.10.14. -Z-DIRECTION. . . . . . . . . . . . . 37

5.7.10.15. +Z-DIRECTION. . . . . . . . . . . . . 37

5.7.11. APPROACH-PALLET Path Point Routines. . . . . . . 38 
5.7.11.1. APPROACH-PALLET. . . . . . . . . . . 38

5.7.11.2. APPROACH-PALLET-INIT. . . . . . . . . 38

5.7.11.3. APPROACH-PALLET-S-M-INIT. . . . . . . . 38

5.7.11.4. CALC-Y-ROT. . . . . . . . . . 38

5.7.11.5. CALC-X-ROT. . . . . . . . . . . . 39

5.7.11.6. CALC-Y-TRANS. . . . . . . . . . . 39

5.7.11.7. CALC-X-TRANS. . . . . . . . . . . . . 39

5.7.12. PICKUP-PALLET Path Point Routines. . . . . . . . 39

5.7.12.1. PICKUP-PALLET. . . . . . . . . . . . . 39

5.7.12.2. PICKUP-PALLET-INIT. . . . . . . . . . . 40

5.7.12.3. PICKUP-PALLET-SONAR-MODEL-INIT. . . . . 40

5.7.12.4. WAIT-RANGE-SET. . . . . . . . . . . . 40

5.7.12.5. MOVE-TO-PALLET. . . . . . . . . . . . 40

5.7.12.6. Z-CORRECTION. . . . . . . . . . . . . 40

5.7.12.7. UPDATE-DISTANCE-TO-GOAL. . . . . . . 41

5.7.12.8. FORK-ALIGNMENT. . . . . . . . . . . 41

5.7.12.9. SWITCH-CORRECTION. . . . . . . . 41

5.7.12.10. SWITCH-READ. . . . . . . . . . . 42

5.7.13. GOTO-UNTIL-SW Path Point Routines. . . . . . . . 42

5.7.13.1. GOTO-UNTIL-SW. . . . . . . . . . . . 42

5.7.13.2. GOTO-UNTIL-SW-INIT. . . . . . . . . . . 42

5.7.14. RETURN-POSE Path Point Routine. . . . . . . . . 42

5.7.14.1. RETURN-POSE. . . . . . . . . . . . . 42

5.7.15. DELAY Path Point Routine. . . . . . . . . . . . 42

5.7.15.1. DELAY. . . . . . . . . . . . . . . 42

5.7.15.2. DELAY-INIT. . . . . . . . . . . . 43

5.8. PATH Postprocessing. . . . . . . . . . . . . . . 43

5.9. Display and Debug Routines. . . . . . . . . . . . . . 43

5.9.1. DISPLAY-SONAR-SELECTION. . . . . . . . . . 43

5.9.2. DISPLAY-PROXIMITY-SWITCHES. . . . . . . . 43 Appendix A Flow Charts

RANGE

EDGE

EQUATE

SCAN

ALIGN-GRIP 
October 4, 1987

APPROACH-PALLET

PICKUP-PALLET 



\section{Introduction}

The National Bureau of Standards is contracted by the United States Army's Human Engineering Laboratory (HEL) to develop a high level sensory interactive robot controller based on the NBS Real-Time Control System (RCS). The controller will be incorporated within the HEL Field Material-Handling Robot (FMR). The primary task of the FMR is the handling of palletized loads; the robot is equivalent to a sensor guided fork lift.

The basic RCS configuration is extensively documented in the NBS Real-Time Control System User's Reference Manual. The following document is the supplement to the RCS Reference Manual. It describes the extensions to RCS that are specific to the FMR. It is assumed that the audience has read the RCS Manual before he or she attempts to read this manual. In any case references to the RCS Manual are given and the RCS Manual table of contents and index should also provide some assistance.

The Robot Sensor Language (RSL), developed at NBS for use with the FMR Real-Time Control System, features sensory interactive off-line (pre-planned) programming. An in depth description of RSL is also presented in the RCS Reference Manual. Several Path Point commands were developed to make use of the FMR sensor package and to program the type of tasks NBS envisioned the FMR would be expected to perform.

Four sections are covered in this document: the sensor modelling techniques used in conjunction with the NBS sensor package, the FMR Path Point commands, two RSL programs used for transferring $155 \mathrm{~mm}$ pallets, and the real-time PATH level code that executes the Path Point commands.

\subsection{Documentation Conventions}

This document uses bold face text to distinguish between program (software).statements and text. Program statements include names of routines, variables and commands that can be entered from a terminal into the RCS. One exception is the bold face used to highlight section numbers and titles.

The syntax for the path point commands in Section 2 use the vertical bar to separate parameter fields.

The FORTH programming language supports some documentation conventions; primarily the left and right parenthesis to bound comments and the percent sign to indicate the remaining portion of the line is a comment. 


\section{Sensor Modelling}

The NBS Real-Time Control System for the FMR utilizes two types of sensors: Acoustic sensors (ultrasonic sonars) provide range data while optical emitter/detector pairs provide proximity information. (Detail information on the sensors can be found in the NBS GFE Technical Data Package for the FMR May 1986.) Two modelling schemes were developed which allow the RCS controller to effectively use the sensors. One scheme models each of the ultrasonic ranging devices. The second scheme models the relationships between the proximity detector configurations and the pallets. The following sections describe the modelling and calibration techniques for each sensor.

\subsection{Sonar Modelling}

The ultrasonic sonars used in the FMR sensor package have been characterized through a process of benchmark testing and live usage. The results of the characterization are embedded in a model used by the control system. The model is stored as a data structure within the RCS file system. The primary benefit of such a scheme is the separation of data from control procedures. Previously the sonar characteristics were stored inside the control level or were entered by the user as parameters within a Path Point command. Any changes to the sonars required modification of the control level or having the user perform some calculation and entering the results through the command call. With the sonar model, any changes only require modification of the model represented in the data structure. The PATH control level interprets the model for data necessary in the execution of control functions such as sensor guided translations and rotations.

\subsubsection{Model Data for the Sonars}

The model for each sonar consists of the following data stored as fields in a RCS record structure.

snr-name

snr-id

snr-type

snr-axis

snr-mtb

snr-angle

snr-min-range The minimum effective range of the sonar.

snr-max-range The maximum effective range of the sonar.

\subsubsection{Compiling the Sonar Model}

The RSL level word -snr- compiles the model. The first step is to edit a block from the RSL level to include all the parameters associated with a sonar. For example: 
-snr-
snr-0
0
Polaroid
$\mathbf{X}$
snr0-mtb
10.0
12.5
128.0

The next step is to load the block which compiles the parameters into the following record and file:

50 bytes VAR-O snr-model-para

$\begin{array}{ll}\text { 6 strv } & \text { "snr-name" } \\ \text { iv } & \text { snr-id } \\ \text { iv } & \text { snr-type } \\ \text { iv } & \text { snr-axis } \\ \text { iv } & \text { snr-mtb-^ } \\ \text { fv } & \text { snr-angle } \\ \text { fv } & \text { snr-min-range } \\ \text { fv } & \text { snr-max-range }\end{array}$

20 rec FILE SONAR-MODEL-FILE

\subsubsection{Interpreting the Sonar Model}

Once the model is compiled into the RCS file system, the PATH level can interpret the SONAR-MODEL-FILE file. To facilitate retrieving the record of a sonar the PATH level maintains an array of pointers, snr-model-array, which maps a physical sonar number $(0$ thru 15$)$ into a record in the file SONAR-MODEL-FILE. The routine INIT-SNR-MODEL-ARRAY maintains the array and is executed at power up. An element in snr-model-array that contains a $0 \#$ indicates the sonar does not exist. The user can retrieve the record of a sonar from within a routine in the following manner:

SONAR-MODEL-FILE \% set file snr-model-array $\{$ sonar\# $\}=>$ record\# \% set record\# with desired pointer retrieve \% retrieve model record

An example of how to use the sonar model can be found in the EQUATE Path Point command routines located on the PATH level (section 5.7.8.3. EQUATE-SONAR-MODEL-INIT).

\subsubsection{Calibrating the Sonar Model}

The only calibration required for the sonar model is the position of the transducer. A movetable is used to define the position of the sonar relative to the tool coordinate frame. The location of the tool frame is specified by the wrist frame and an added tool movetable called TOOL-MTB. The tool frame is specified by the programmer and typically only changes with the end-effector. If the tool frame is changed, then an appropriate change must also be made in the sonar movetables.

\subsection{Proximity Detector Modelling}

The proximity detectors for the FMR are used to prevent the fork tines from colliding with the pallet support structures (feet) during insertion. Eight detectors are positioned at the tip of FMR fork tines (see Figure 2.1.). Figure 2.1. shows the relationship between the fork and a $155 \mathrm{~mm}$ pallet support structure prior to insertion (perspective view). The top view shows the desired alignment between the fork and the structure. An enlargement of that view illustrates the relationship between the left 
tine with it's proximity detectors and the pallet foot (right tine is a mirror image with detectors 4-7). Note that the proximity detector's field of view is very narrow (1-3 degrees) and the sensitivity of the detector allows them to pickup obstacles at approximately 4-6 inches (this is adjustable).

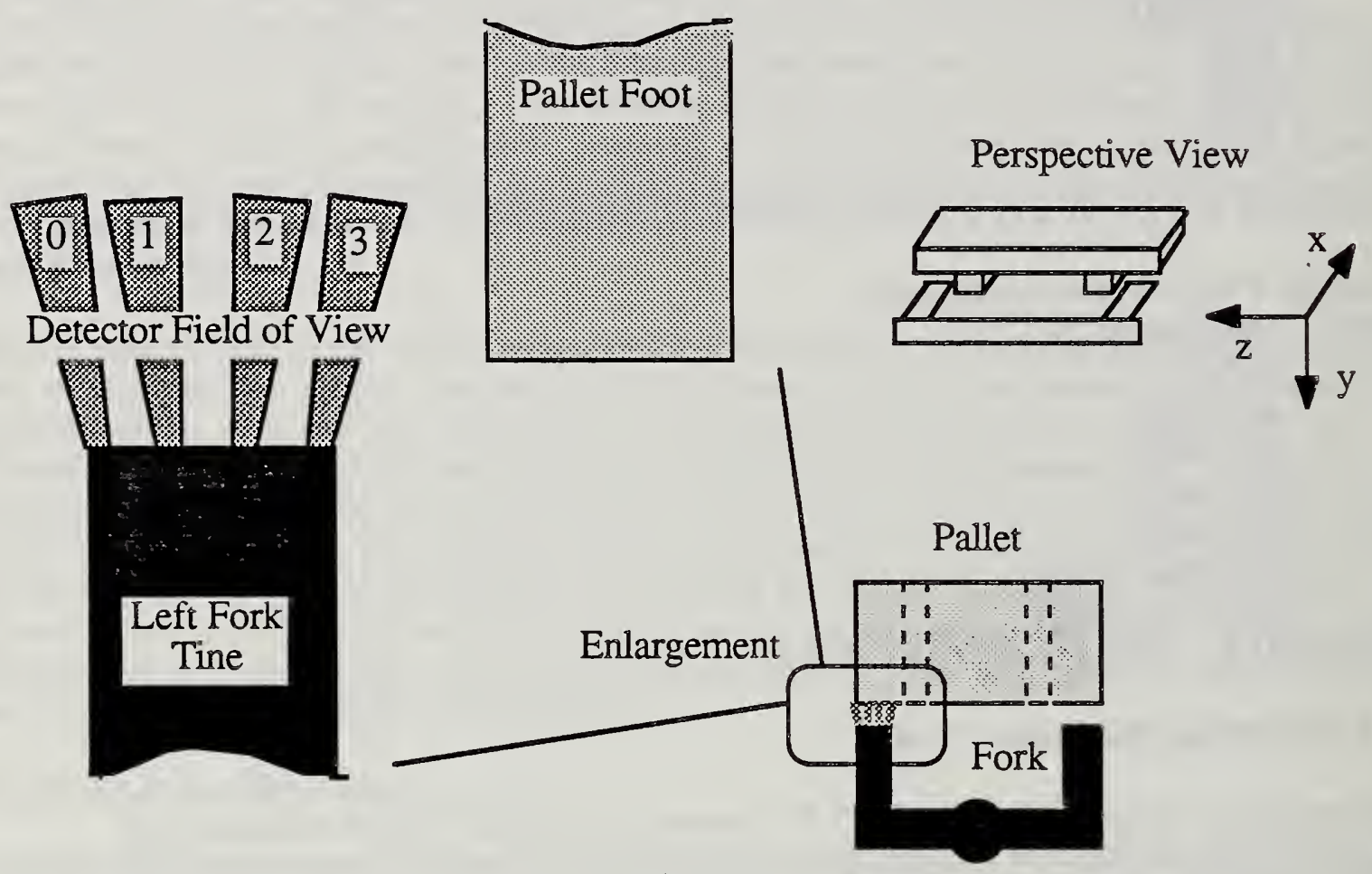

Figure 2.1. The relationship between the fork proximity detectors and a $155 \mathrm{~mm}$ pallet.

The proximity detector configuration allows the FMR to correct a misalignment (translation along the $\mathrm{Z}$ axis of the tool frame) of approximately plus/minus six inches from the ideal insertion position.

Figure 2.2 shows an example of how the proximity sensors 1,2 and 3 detect a misalignment in the $-Z$ direction. 

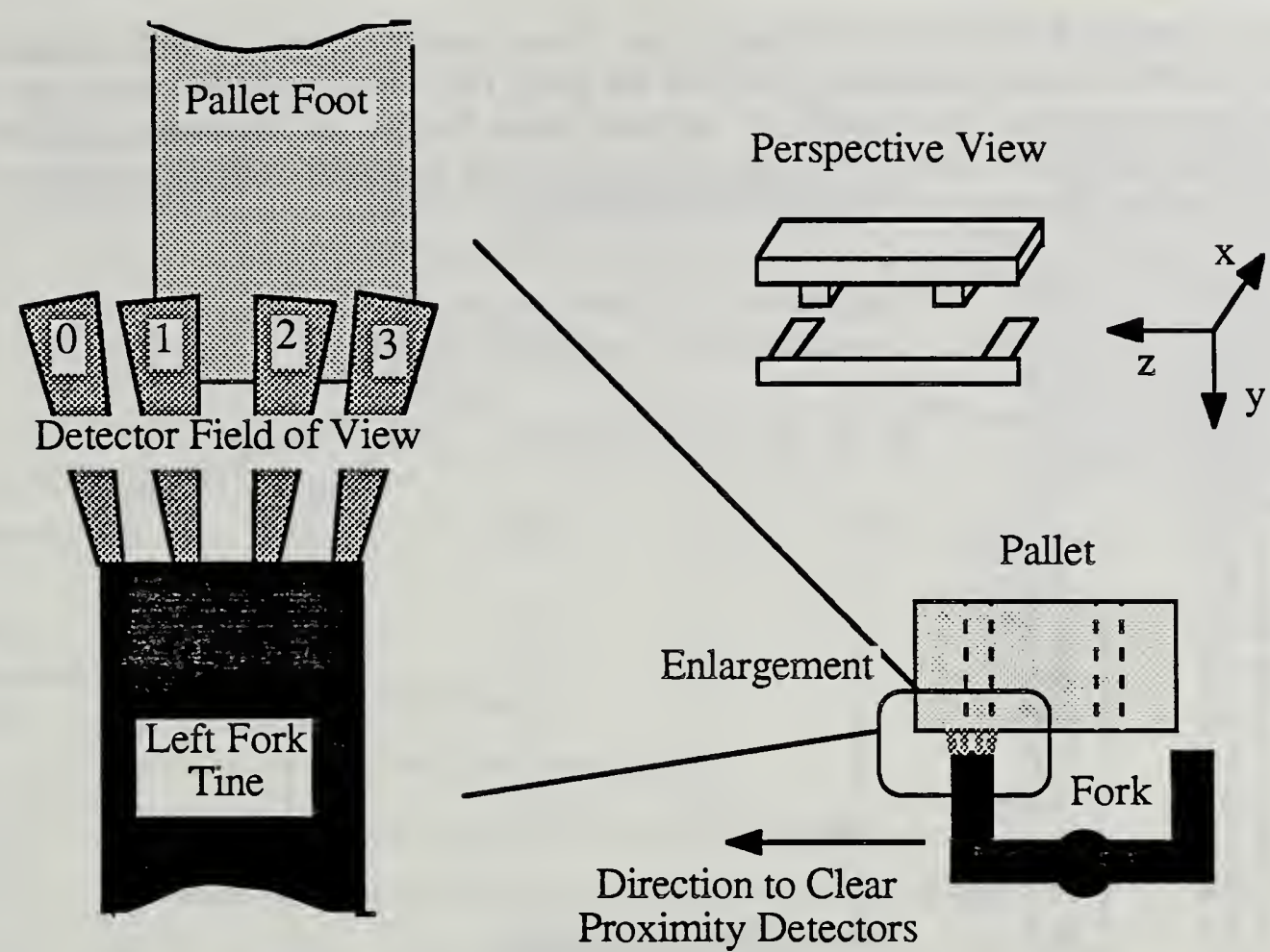

Figure 2.2. The Proximity sensors detecting a misalignment between the fork and pallet.

Detector 0 limits the worst case misalignment ( 6 inches) that the FMR can correct in the $-Z$ direction. The Path Point command pickup-pallet uses the proximity detector configuration to correct misalignments (sections 3.7. and 5.7.12.).

The modelling for the detectors uses a simple technique which optimizes run-time execution by compressing as much information as possible into the model in an off-line fashion. The technique is to compile a look-up table which directly maps an active detector into the necessary motion to clear the obstacle. For the case of the $155 \mathrm{~mm}$ pallets the look-up table is generated by placing the pallet in front of the fork tines to block a particular combination of detectors. The pallet is then moved until the detectors are clear from the foot. Finally the movement is measured and stored into the look-up table. This is repeated for all combinations of detector blockages. At run-time the detectors are read and the combination of the active detectors are used to directly access the lookup table and retrieve the required movement to clear the blockage.

\subsubsection{Model Data for the Proximity Detectors}

Two floating point arrays are used to store the look-up tables for the left and right tine proximity detector configurations: l-tine-calib-table and $\mathbf{r}$-tine-calib-table. Each element in the array contains either a value indicating the translation motion for the associated active detectors or an error. Currently the error value is the number 0.0 and the error condition indicates an impossible combination of active detectors. As an example, in the case of the $155 \mathrm{~mm}$ pallet it is impossible for detectors 1 and 3 (see Figure 2.2.) to be active while detector 2 is not. If this does occur it indicates one of the detectors are bad, misaligned or possibly obstructed by dirt. Another possibility is the pallet foot does not measure up to expectations.

\subsubsection{Compiling the Proximity Detector Model}

The two tine calibration tables are edited into a power-up block on the PATH level. The blocks are 
loaded each time the PATH level is brought up. If any modification is made to the table, the block must be reloaded. It is not necessary to save the table (ie. MEM $>$ DISK) since the table is always loaded during power-up. An example of the load block for the left tine table follows. The comments (within parentheses) serve two purposes: first they show the array element number and second they illustrate the active detectors associated with the entry.
$\left(\begin{array}{llll}0 & 0 & 0 & 0\end{array}\right)$
0.0
$\left(\begin{array}{llll}0 & 0 & 0 & 1\end{array}\right)$
8.25
$\left(\begin{array}{llll}0 & 0 & 1 & 0\end{array}\right)$
6.0
$\left(\begin{array}{llll}0 & 0 & 1 & 1\end{array}\right)$
7.0
$\left(\begin{array}{llll}0 & 1 & 0 & 0\end{array}\right)$
4.0
$\left(\begin{array}{llll}0 & 1 & 0 & 1\end{array}\right)$
0.0
$\left(\begin{array}{llll}0 & 1 & 1 & 0\end{array}\right)$
4.875
$\left(\begin{array}{lllll}0 & 1 & 1 & 1\end{array}\right)$
5.75
$\left(\begin{array}{llll}1 & 0 & 0 & 0\end{array}\right)$
1.875
$\left(\begin{array}{llll}1 & 0 & 0 & 1\end{array}\right)$
0.0
$\left(\begin{array}{llll}1 & 0 & 1 & 0\end{array}\right)$
0.0
( $\left.\begin{array}{llll}1 & 0 & 1 & 1\end{array}\right)$
0.0
$\left(\begin{array}{llll}1 & 1 & 0 & 0\end{array}\right)$
3.625
( 11101$)$
0.0
(11 110$)$
3.875
( 111111 )
0.0
$==>$ I-tine-calib-table

\subsubsection{Interpreting the Proximity Detector Model}

The Path Point command pickup-pallet is an example of how the proximity detector model is interpreted. The NBS system currently only supports the model for the $155 \mathrm{~mm}$ pallet. The system can easily be extended to support a model for each different type of pallet. This would require the development of a model compiler word similar to the sonar model compiler -snr-.

\subsubsection{Calibrating the Proximity Detector Model}

The look-up table is generated by positioning the pallet in front of each proximity sensor and moving the pallet until it no longer detects the foot. This measures the field pattern, the position and the orientation of the sensor (with relation to the pallet foot) while also measuring the reflective properties of the pallet surface. The entry into the table is the measurement of the distance the pallet is moved. A plus or minus sign is included to indicate the direction the fork needs to travel to clear the foot. This process must be repeated for each combination of active detectors. The remaining combinations of active detectors that cannot be achieved by positioning the pallet are flagged with a 0.0 indicating a possible error condition.

Achieving each combination of sensors is not difficult. It is recommended that a pallet be placed on wheels so that it can be slowly passed by the detector configuration until there is a change in the combination of active sensors. The change can be seen by viewing the LED indicators on each proximity board in the interface chassis or by using the DISPLAY-PROXIMITY-SWITCHES routine (section 5.9.2.). A sheet of paper taped to the floor is used to mark each change in the detector combination and the position where the change occurred. Once complete the paper is removed and measurements are made from the point on the paper where each combination of detectors were active to the common point where all detectors were inactive. Remember that the measurement must reflect the motion and direction the fork needs to travel to guarantee the detectors become inactive. Figure 2.3 shows a sample sheet generated during the calibration of the left tine proximity detector configuration with a $155 \mathrm{~mm}$ pallet foot. Each occurrence of a change in active detector combinations is recorded while the pallet is moved from left to right. The active detectors are shown starting with a 0000 to indicate all detectors are inactive. Detector 0 is the first to see the pallet 
and detector 3 is the last.

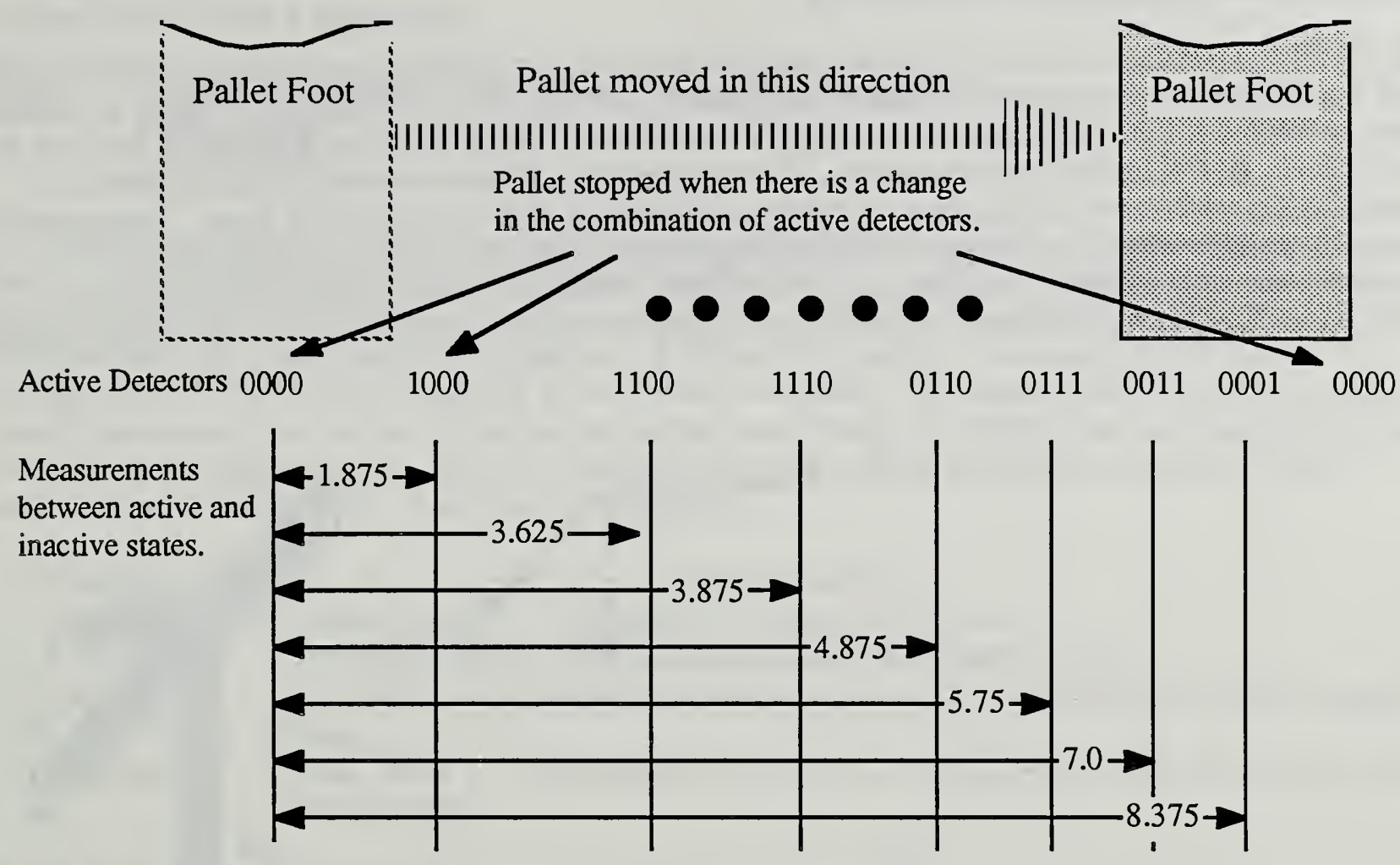

Figure 2.3. Example calibration of left fork tine proximity detector configuration. 


\section{Path Point Commands for the FMR}

The FMR Path Point commands give the programmer the capability to position and orient the FMR fork end-effector using sensor feedback supplied by the FMR sensor package. Figure 3.1 shows a fork mockup with the NBS sensor package. A coordinate frame is shown attached to the fork and is useful for specifying tool based motions. For the purpose of this document, it is sufficient to visualize the position of the frame as between and parallel with the tips of the tines. The package currently consists of eight Polaroid and five Migatron ranging devices, and eight optical emitter/detector proximity switches (the NBS FMR Technical Data Package, Section 5.6.1. SONAR-READ and Section 2.Sensor Modelling give further information on the sensor package).

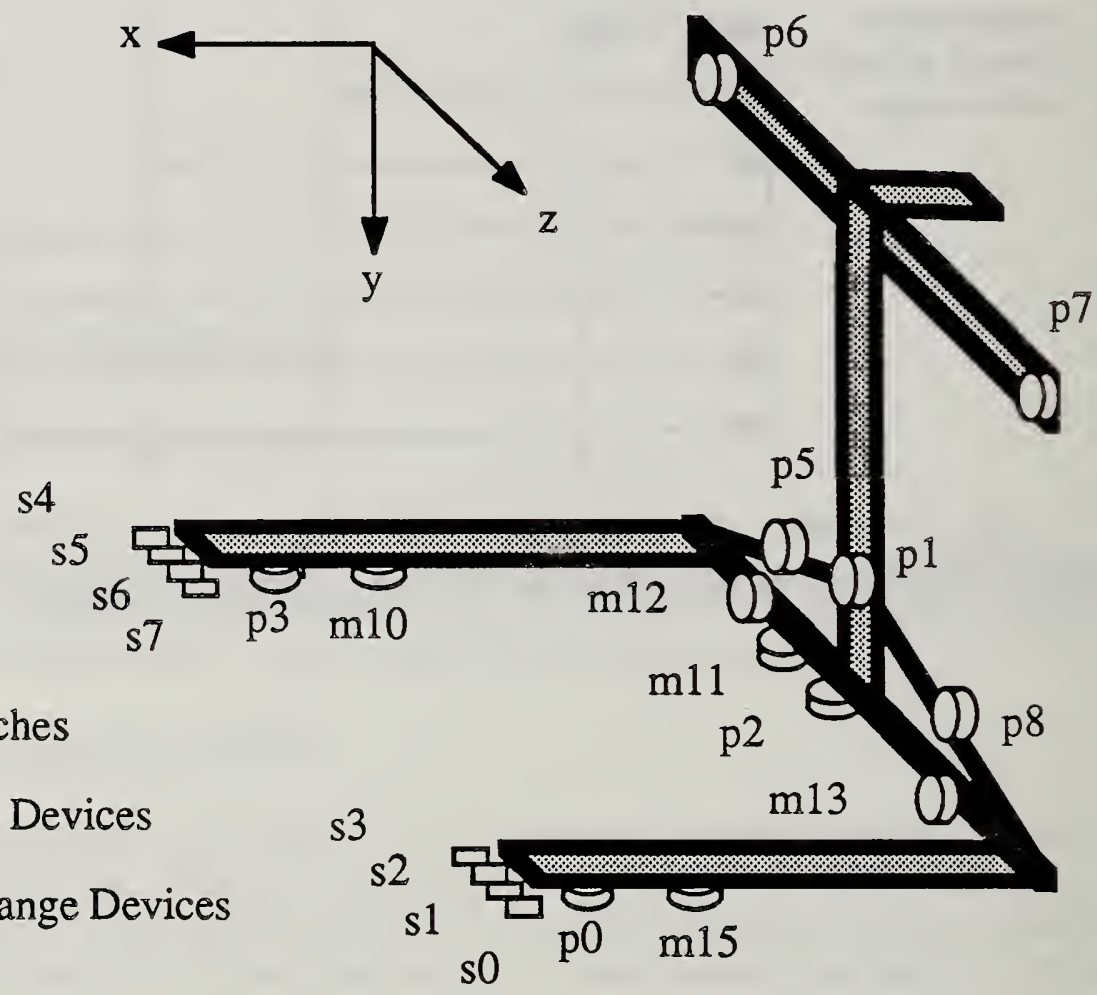

Figure 3.1. The FMR Sensor Package with Tool Coordinate Frame

The Path Point commands can be combined in a logical sequence to perform such tasks as aligning the fork with a truck bed, locating a randomly oriented pallet and transferring an array of pallets. Briefly the range and edge commands provide translation and pallet recognition capabilities; equate allows the fork to be rotated and aligned; scan, align-grip, approach-pallet, and pickup-pallet are specialized commands for locating a $155 \mathrm{~mm}$ pallet, determining the pallet entry side, aligning and preparing the fork for insertion, and inserting the tines respectively. Some additional commands are also included. A description of the Path Point commands and their syntax is discussed next.

The parameters for a Path Point command vary but two types of parameters are supported by the base RSL system: the Trajectory Phrase and the Location Phrase. In this section, the words traj phrase and loc phrase are used for the Trajectory Phrase and the Location Phrase respectively. Their specific format is explained in the RCS Manual (Appendix B-70). The reader can look ahead to Section 4 for examples of the usage of Path Point commands in the context of an RSL program. The 
specific commands are now examined.

\subsection{Range Path Point Command}

\begin{tabular}{|c|c|}
\hline range & $\begin{array}{l}\text { r-sonar | r-range | r-threshold | r-axis | halt-enable } \\
\text { loc phrase | } \\
\text { trai phrase | }\end{array}$ \\
\hline
\end{tabular}

The range command is used to position the fork a specified range from a target. The parameters specify a move along the r-axis axis of the cartesian frame specified by the loc phrase (only tool nul frame is supported) to the range measured by the sonar $r$-sonar\#, within $\mathbf{r}$-threshold. The commanded translation is positive if $\mathbf{r}$-range - $\mathbf{r}$-sonar\#\{range-value $\}>0$ (\{range-value $\}$ is the range returned by the sonar r-sonar\#). A sonar-limit error is returned if a range is reported below that of the the minimum sonar range detectable (the minimum is derived from the model of the sonar). This can occur if the wrong sonar number is entered or if the wrong sonar is selected (ie. Polaroid vs. Migatron).

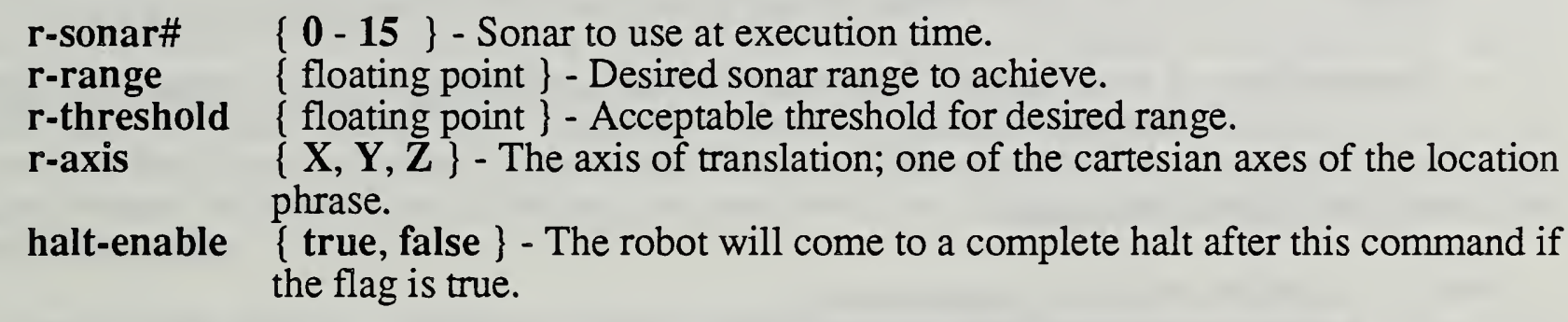

\subsection{Edge Path Point Command}

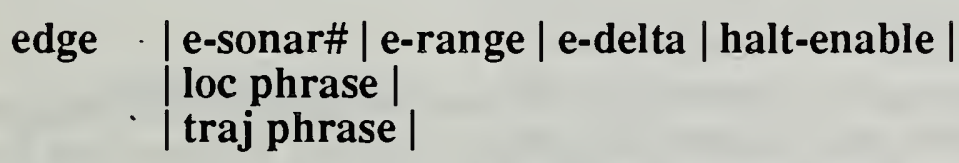

The edge command is used to locate edges. An edge is defined as a difference in range readings of at least e-delta. The set of range readings bounded by e-delta must also include the value specified in e-range. In other words e-delta describes the magnitude of the edge and e-range declares at what distance to search for it (see Section 5.7.7.4. EDGE). If the halt-enable flag is true the system initiates a halt when the sonars first satisfy the edge conditions. The command does not servo to the sensor condition. If the sonar condition is not satisfied after the halt is completed (due to overshoot, for example), the error sensor-cond is reported. If the robot reaches the location before the sonar condition is satisfied the error point-reached is reported. The loc phrase is the robot goal and is used to specify where to search for the edge. The traj phrase is used to specify the type of motion. A joint trajectory allows fast searches of large areas. A cartesian trajectory will force the fork to travel in a straight line.

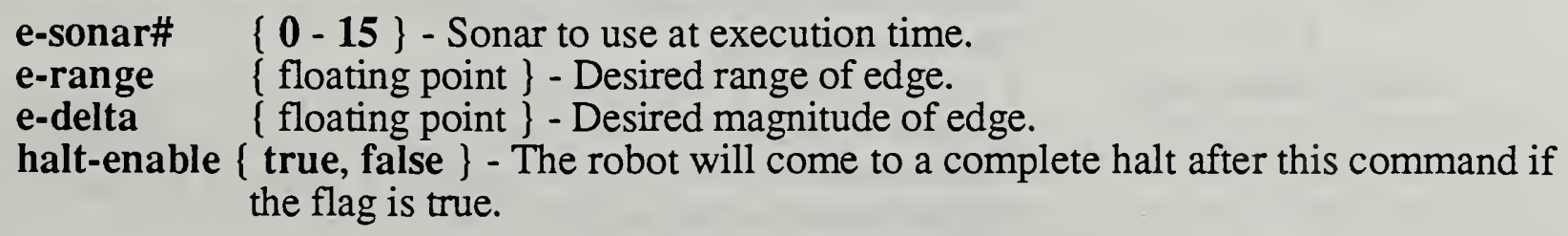
the flag is true.

\subsection{Equate Path Point Command}

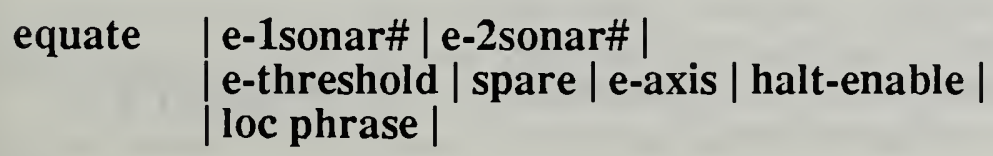




\title{
| traj phrase |
}

The equate command is used to rotate the fork to achieve balanced range readings between the two sonars, e-1sonar\# and e-2sonar\#. The parameters command a rotation about the e-axis axis of the cartesian frame specified by the loc phrase (only the tool nul frame is supported) until the two selected sonars read the same, within e-threshold. Since the rotation commanded will be positive about an axis if sonar\#1 \{range-value - sonar\#2\{range-value $>0$ it is necessary to consider the order of the selected sonars. As an example if the programmer desired to use a pair of down-looking sonars to align with the floor, sonars 0 and 3 are used to command a rotation about the $\mathrm{X}$ axis (see Figure 3.1.). To achieve the proper direction parameter sonar\#1 is set to 3 and sonar\#2 is set to 0 .

sonar\#1
sonar\#2
threshold
spare
axis

halt-enable
$\{0-15\}$

$\{0-15\}$ - The two sonars to equate.

\{ floating point - Acceptable threshold for balanced sonars.

$\{0.0\}$ - Not used.

$\{\mathbf{X}, \mathbf{Y}, \mathbf{Z}\}$ - The axis of rotation; one of the cartesian axes of the tool frame.

\{true, false - The robot will come to a complete halt after this command if the flag is true.

\subsection{Scan Path Point Command}

\author{
scan | s-select | skip-till | pallet-area | \\ closest-point-delta | spare | scan-return-pose- ${ }^{\wedge} \mid$ halt-enable | \\ loc phrase | \\ |traj phrase |
}

This is a high level Path Point command used to determine the nominal location of a rectangular object such as a $155 \mathrm{~mm}$ pallet. The robot scans an area taking range readings starting from it's present position and ending at loc phrase. Each range reading is linked to the position of the robot when the sonars are read and then stored into the file SONAR-FILE. The scan command returns the nominal pose of the object in the record pointed to by scan-return-pose-^ (Section 5.7.9.12. RETURN-SCAN-POSE describes the nominal pose of a pallet). If a pallet is not found the error no-pallet is returned.

\section{s-select \\ skip-till}

pallet-area

closest-point-delta

spare

scan-return-pose-^
$\{0$ - 15$\}$ - Sonar to use at execution time.

\{ integer \} - Skip this number of valid sonar readings before storing the value with the robot pose. This conserves the number of readings taken but reduces the resolution of the scan.

\{ floating point \} - Used as the maximum range where the pallet can be from the sonar. The first and last readings of the scan less then max-object-range determine the right and left edge of the pallet respectively.

\{ floating point \}- Sets the delta of range values that can be grouped together with the closest range. This group then provides information such as whether a side or a corner of the pallet is the closest feature of the pallet to the robot (See 5.7.9.12.

RETURN-SCAN-POSE).

$\{0\}$ - Not used.

\{ Ascii string - Names a pose which scan sets to the nominal pose of the pallet as derived from the search. The parameter is the name of the pose, the compiler searches the POSE-FILE and stores the record\# of the pose in the parameter. An error will be 
halt-enable returned if the pose has not been previously defined. \{true, false \} - The robot will come to a complete halt after this command if the flag is true.

\subsection{Align-grip Path Point Command}

align-grip

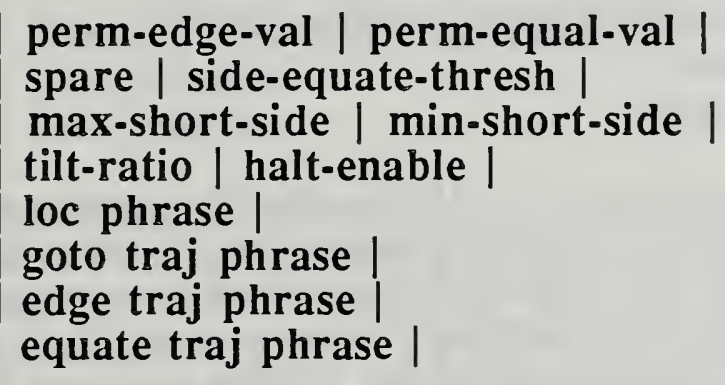

This is a high level Path Point command which utilizes information from scan and current sonar data to determine if the long side of a $155 \mathrm{~mm}$ pallet is facing the robot. It is assumed the fork is within 25 inches of the pallet and the orientation of the $\mathrm{YZ}$ plane of the fork with respect to the pallet falls into one of three cases: Long-case where the fork faces the pallet long side, Short-case where the fork faces the pallet short side and Tilt-case where the fork faces a corner of the pallet. Edge, equate and goto type commands are performed to roughly align the fork with the long side of the pallet.

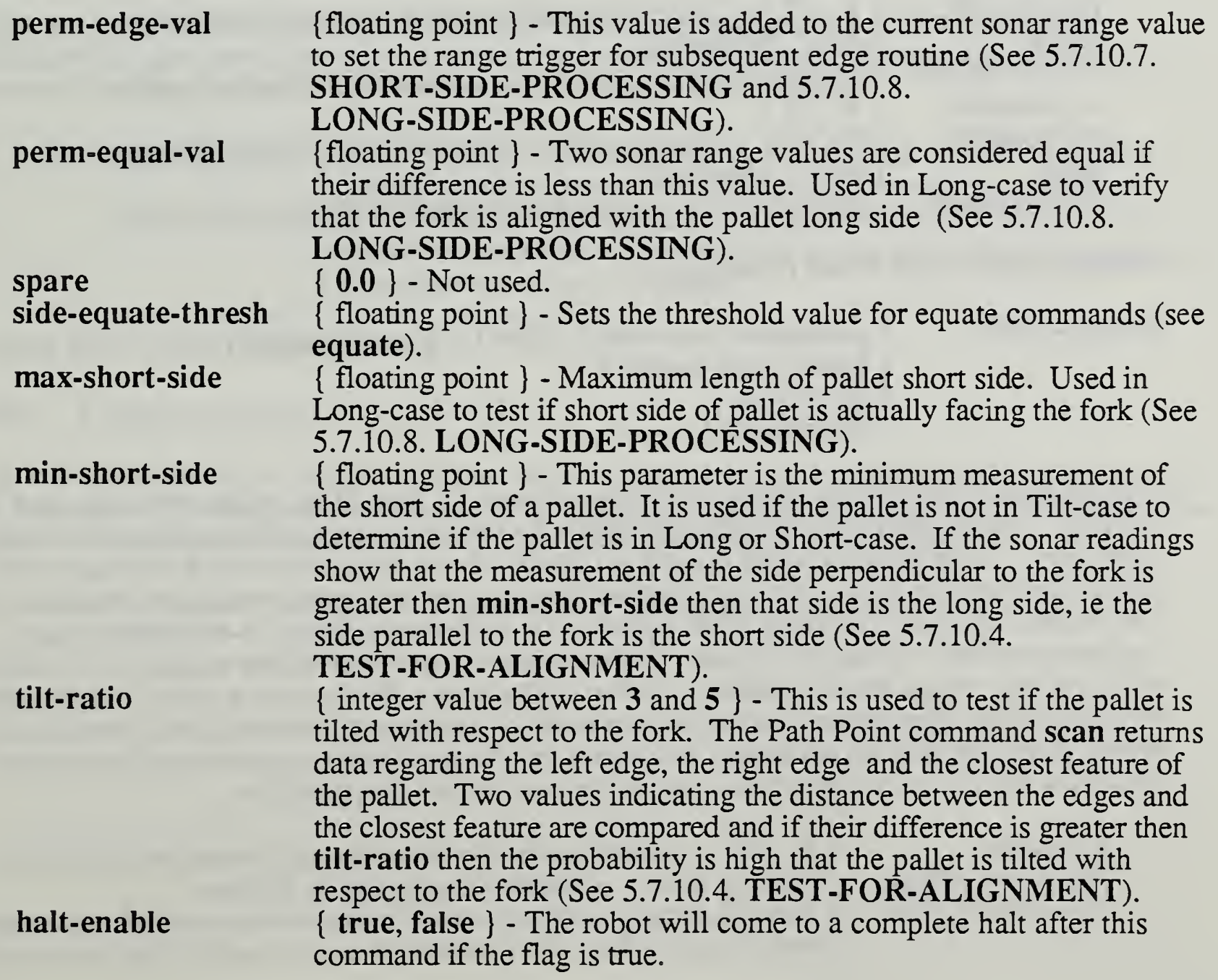




\title{
3.6. Approach-pallet Path Point Command
}

\begin{tabular}{|c|c|c|}
\hline pproach-pallet & \begin{tabular}{l|l} 
t-x-sonar\# & $t-x-$ range \\
t-y-sonar\# & t-y-range
\end{tabular} & $\begin{array}{l}\text { t-x-threshold } \\
\text { t-yothreshold }\end{array}$ \\
\hline & |r-x-sonar\#2 | spare & $\mathbf{r}=\mathbf{X}$-threshold \\
\hline r-y-sonar\#1 & $\begin{array}{l}\mid \mathbf{r}-\mathbf{y} \text {-sonar\#2| } \\
\text { loc phrase | } \\
\text { traj phrase | }\end{array}$ & roy-threshold \\
\hline
\end{tabular}

This command handles the final approach to the $155 \mathrm{~mm}$ pallet long side by achieving the proper height and orientation of the fork prior to inserting the tines beneath the pallet. Four tool motions are calculated in a manner similar to the range and equate Path Point commands. Ranges are specified by the $\mathbf{t}-\mathbf{x}$ - and $\mathbf{t}-\mathbf{y}$ - parameters signifying translation along the $\mathrm{X}$ and $\mathrm{Y}$ axes. Equates are specified by the $\mathbf{r}-\mathbf{x}$ - and $\mathbf{r}-\mathbf{y}$ - parameters signifying rotation about the $\mathrm{X}$ and $\mathrm{Y}$ axes. The parameters are the same as the parameters for range and equate.

\begin{tabular}{|c|c|}
\hline t-X-sonar\# & 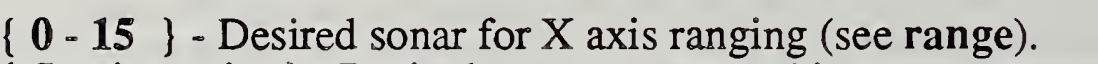 \\
\hline$t=x-$ range & floating point \} - Desired sonar range to achieve. \\
\hline toX-threshold & floating point $\}$ - Acceptable threshold for desired range. \\
\hline t-y-sonar\# & 0 - 15 $\}$ - Desired sonar for $\mathrm{Y}$ axis ranging (see range). \\
\hline t-y-range & floating point \} - Desired sonar range to achieve. \\
\hline $\begin{array}{l}\text { t-y-threshold } \\
\text { r-X-sonar\#1 }\end{array}$ & $\begin{array}{l}\text { floating point }\} \text { - Acceptable threshold for desired range. } \\
0-15\}\end{array}$ \\
\hline $\begin{array}{l}\mathrm{r}-\mathrm{X}-\mathrm{sonar} \# 2 \\
\text { sDare }\end{array}$ & $\begin{array}{l}0 \text { - 15 - Desired sonars for } \mathrm{X} \text { axis rotation (see equate). } \\
0.0\end{array}$ \\
\hline $\begin{array}{l}r \cdot x \text {-threshold } \\
r=y=\text { Sonar\#1 }\end{array}$ & floating point - Acceptable threshold for balanced sonars. \\
\hline $\begin{array}{l}r \text {-y-sonar\#2 } \\
\text { spare }\end{array}$ & $\left\{\begin{array}{l}0-15 \\
0.0\end{array}\right\}$ - Not used. \\
\hline r-y-threshold & \\
\hline
\end{tabular}

\subsection{Pickup-pallet Path Point Command}

\author{
pickup-pallet |p-p-sonar | p-p-sonar-offset | p-p-z-correction | \\ | spare | halt-enable | \\ loc phrase | \\ | traj phrase |
}

This command insures that the robot fork tines do not hit the $155 \mathrm{~mm}$ pallet feet as the fork is inserted. Optical proximity sensors (see Figure 3.1 ) detect when the tines are too close to the pallet feet. The p-p-sonar sonar is used to initially obtain the distance the fork must travel before stopping the robot. The programmer can use the parameter p-p-sonar-offset to modify the distance the fork will travel to the pallet in the following manner: p-p-sonar\#\{value $\}$ p-p-sonar-offset $=$ distance to pallet. For example if $\mathbf{p}$-p-sonar-offset equals 4.0 , the fork will stop four inches from the pallet. When a proximity sensor senses a pallet foot the robot translates in the appropriate $Z$ axis direction until the sensor is no longer active. Because of the sensor characteristics an additional translation can be specified in p-p-Z-correction which forces the fork to travel further after the sensor has cleared the pallet foot.

p-p-sonar

p-p-sonar-offset p-p-z-correction
\{ 0 - 15$\}$ - Desired sonar for determining distance to pallet load. \{ floating point \} - Desired sonar range to achieve.

\{ floating point \} - Desired $Z$ axis motion to be travelled after sensor clears the pallet foot. This parameter can be used to help center the fork 
since there may be a large dead-band where none of the fork tine proximity sensors can detect the pallet feet.

spare $\{0.0\}$ - Not used.

\subsection{Goto-until-sw Path Point Command}

goto-until-sw

$$
\begin{aligned}
& \text { | desired-sw | desired-sw-status | } \\
& \text { loc phrase | } \\
& \text { traj phrase | }
\end{aligned}
$$

The goto-until-sw command operates similarly to the goto Path Point command except the motion is terminated if the selected switch specified by desired-sw achieves the desired condition as indicated by desired-sw-status. The error point-reached is returned if the robot reaches the goal specified by loc phrase prior to satisfying the switch condition.

switch

$\{0$ - 7$\}$ - The desired switch. Currently eight switches on the fork tines can be used. Additional switches can be integrated, up to sixteen total.

switch condition $\quad\{$ open, closed \} - The desired switch condition.

\section{9.. Return-pose Path Point Command}

\section{return-pose | return-pose-para-^ |}

This command sets the pose record specified by return-pose-para-^ (defined in the data dictionary) to the current pose of the robot. This command is used to dynamically store a pose after the robot has moved to a position (usually under sensor control).

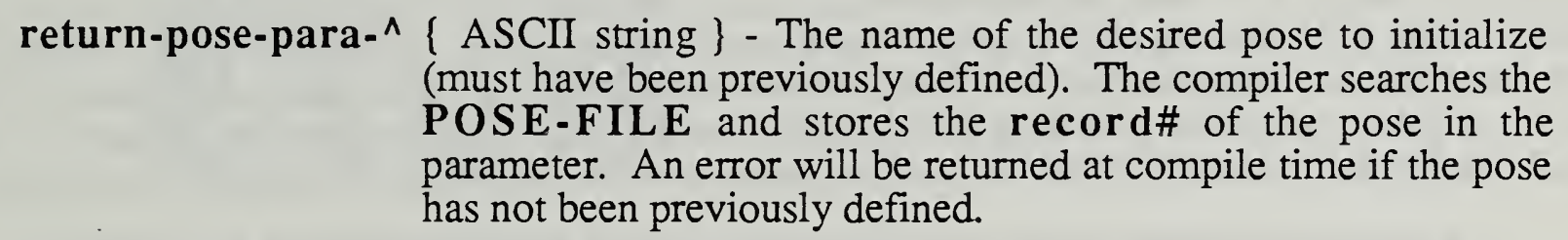

\section{9.. Delay Path Point Command}

\section{delay | delay-\#-cycles |}

This command is used to introduce delays between the execution of other Path Point commands. An example of it's usage is to allow the fork to stabilize during complete halts. delay-\#-cycles specifies the number of control cycles to delay before terminating the command.

delay-\#-cycles \{ integer $\}$ - The number of control cycles to delay. 


\section{Programming the FMR Using RSL}

The primary command for the FMR is TRANSFER. The TRANSFER command is decomposed by the TASK level into six steps which specify the paths for the pickup and release of an object. All six paths must be defined and compiled from the RSL level (RCS Manual Chapter 10 pages 10-8 thru 10-10) before the TRANSFER command is executed. The six paths are:
1) move-to
2) approach-pickup
3) depart-pickup
4) move-to
5) approach-release
6) depart-release

Two examples of RSL programs (consisting of the six paths) are now given. The first is a plan to locate and transfer a randomly oriented pallet from a truck bed to a conveyor. The second example is a plan to locate and transfer an array of pallets from a truck bed to a conveyor. A randomly oriented pallet has no constraints on which side of the pallet faces the robot and therefore the controller must determine the proper entry side. The array of pallets is constrained such that it's orientation does not vary from a predefined orientation by greater then 10 degrees and the entry side of each pallet within the array is known apriori.

\subsection{Transfer of a Randomly Located $155 \mathrm{~mm}$ Pallet}

\subsubsection{The RSL Plan for Random Pallet}

The plan to transfer a $155 \mathrm{~mm}$ pallet assumes a pallet is randomly positioned on a truck bed and the location of the truck and the conveyor have been taught before hand. Briefly the plan commands the robot controller to: align the fork with the truck bed, search for a $155 \mathrm{~mm}$ pallet, determine the entry side of the pallet, insert the fork tines and finally place the pallet on the conveyor. Figure 4.1. shows a truck loaded with several pallets; the random pallet scenario handles only one pallet on the truck.

( This path moves the robot to a starting position before scanning the truck.) -path- move-to PALLET I loc HOME loc TRUCK

( Go to a predefined location where the truck bed will be using a fast joint trajectory motion.)

-ppt- goto goal nul

joint 30.030 .05 .0

( Achieve proper roll orientation.)

-ppt- equate $0 \begin{array}{llll}0 & .5 & .0 & \mathrm{X} \\ \text { true }\end{array}$

tool nul

cart $\quad .2 .25 .25$

(Achieve proper pitch orientation.)

-ppt- equate $30.520 .0 \mathrm{Z}$ true

tool nul

$\begin{array}{lllllllll}\text { cart } & .2 & .15 & .25 & .25 & .25\end{array}$

(Achieve proper elevation from truck bed.)

-ppt- range 0 18.00 .50 Y true

tool nul 
( This path scans the truck from left to right searching for a single pallet, aligns the fork with long side of pallet, and engages the fork.)

-path- approach-pickup PALLET 1 loc TRUCK

( Search for the pallet using a joint trajectory with SCAN-MTB defining the destination of the search.)

-ppt- scan $\quad 0 \quad 0 \quad 50.0 .50$ PALLET-POSE true tool SCAN-MTB

joint 25.025 .05 .0

( Scan has returned the nominal position of the pallet in PALLET-POSE. Now go to the location PALLET-LOC which is defined as the pose

PALLET-POSE with an offset to safely position the fork in front of the pallet.)

-ppt- goto loc PALLET-LOC joint 25.025 .01 .0

(Make sure fork is within 25 inches of pallet before executing align-grip.)

-ppt- range $120.001 .0 \mathrm{X}$ true

tool nul

cart $.2 .15 .25 .3 \quad 2.25$

( Align with the pallet long side.)

-ppt- align-grip

$\begin{array}{lllllllll}4.0 & 8.0 & 0.0 & .75 & 15.0 & 14.0 & 5\end{array}$

true tool nul

cart $.3 .15 .25 \quad .3 .15$. 15

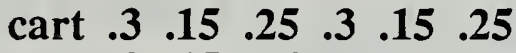

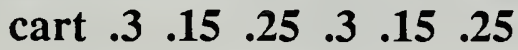

(Lower fork so that the lower base of the pallet acts as the sonar target.)

-ppt- range $152.00 .25 \mathrm{Y}$ true tool nul

cart .2 $2.15 \quad 25.3 \quad 2.25$

(Achieve fine alignment with pallet entry side)

-ppt- approach-pallet

$7 \quad 17.75 \quad .5$

$\begin{array}{lll}15 & 28.5 & .25\end{array}$

1015.0 .25

$\begin{array}{lllll}7 & 6 & .0 & .5 & \text { true }\end{array}$

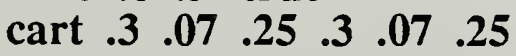

( Guide fork underneath the pallet until within range of sonar 12.)

-ppt- pickup-pallet $74.0 \quad 0.5$ true

cart .3 .3 .25 .3 .25

( Use sonar to achieve precise servoed distance.)

-ppt- range $123.0 .25 \mathrm{X}$ true

tool nul

$\begin{array}{llllllllll}\text { cart } & .2 & .25 & .25 & .25 & .25\end{array}$ 
( Move in the final distance to engage pallet.)

-ppt- goto tool PALLET-ENGAGE

cart $.3 \quad 3 \quad .35 .3 .3 .25$

( This path departs the from the truck by picking up the pallet and tilting it back)

-path- depart-pickup PALLET 1 loc TRUCK

( Goto PALLET-UP1 which is an offset up from current position under pallet.)

-ppt- goto tool PALLET-UP1

$\begin{array}{lllllllll}\text { cart } & .3 & .3 & .25 & .3 & .3 & .25\end{array}$

( PALLET-UP2 tilts pallet back.)

-ppt- goto tool PALLET-UP2

cart .3 .3 .25 .3 .3 .25

( The second move-to path describes a movement to an intermediate safe position.)

-path- move-to PALLET 1 loc TRUCK loc CONVEYOR

( CONV-SAVE is predefined safe position near the conveyor which can be approached using a high joint velocity.)

-ppt- goto loc CONV-SAFE

joint 10.030 .05 .0

( This path describes the approach to the conveyor and the release of the pallet. CONVEYOR location has been taught beforehand.)

-path- approach-release PALLET 1 loc CONVEYOR

( CONV-UP1 is an offset up from CONVEYOR goal. Remember the goal type takes the movetable, CONV-UP1, and adds it to the path goal,

CONVEYOR.)

-ppt- goto goal CONV-UP1

joint 10.030 .05 .0

(CONV-UP2 removes the tilt of the pallet with respect to the CONVEYOR.)

-ppt- goto goal CONV-UP1

cart $.3 \quad 3 \quad 25.3 .3 .25$

(Place the pallet at the goal: CONVEYOR.)

-ppt- goto goal nul

cart $.3 \quad 3 \quad .25$. 3.3 .3

( Extract the fork tines.)

-path- depart-release PALLET 1 loc CONVEYOR

( CONV-BACK extracts the fork from the CONVEYOR.)

-ppt- goto goal CONV-BACK

joint 10.030 .05 .0 


\subsubsection{The RSL Command to Transfer a Random Pallet}

The command to transfer the pallet based on the plan described in Section 4.1.1. has the following syntax:

\section{TRANSFER PALLET 1 loc TRUCK loc CONVEYOR}

The command is entered from the TASK level (RCS Manual Chapter 9.4, page 9-7). The robot must currently be at the location HOME or a nul-path error will be generated (unless another path is mistakenly found).

\subsection{Transfer of a Randomly Located Array of $155 \mathrm{~mm}$ Pallets}

\subsubsection{RSL Plan to Transfer an Array of Pallets}

The RSL plan to transfer an array of pallets illustrates some concepts of the NBS controller. The first concept is that of the array. The array is a data structure defined by the programmer from the RSL level (RCS Manual Chapter 10). For the FMR the array allows a collection of pallets to be organized as sectors in a structured manner.

The second concept is how the TASK level interprets the array structure during the execution of the TRANSFER command. The TRANSFER command syntax includes a field (the sector list) that allows the programmer to specify which pallets (sectors) and in what order the pallets (sectors) are to be transferred. If more than one pallet is to be transferred the Task level will step through the array. Each step through the array consists of using the array movetables, which give the dimensions of each sector, to re-position the fork between the transfer of each pallet.

Figure 4.1. depicts the scenario associated with the array transfer plan. A partially filled $2 \times 3$ array (sectors 1 and 5 are empty) is randomly situated on a truck bed. The FMR searches for sector 0 of the array. This sector is used to define the position and the orientation of the array. As stated earlier, all other sectors are related to the array defining sector by the array movetables. The plan takes into account minor deviations in position (plus/minus six inches) and orientation (plus/minus five degrees) in the placement of a pallet withing a sector. A more sophisticated plan would handle worse alignments but this results in increased sensor processing and consequently longer transfer cycle times. 


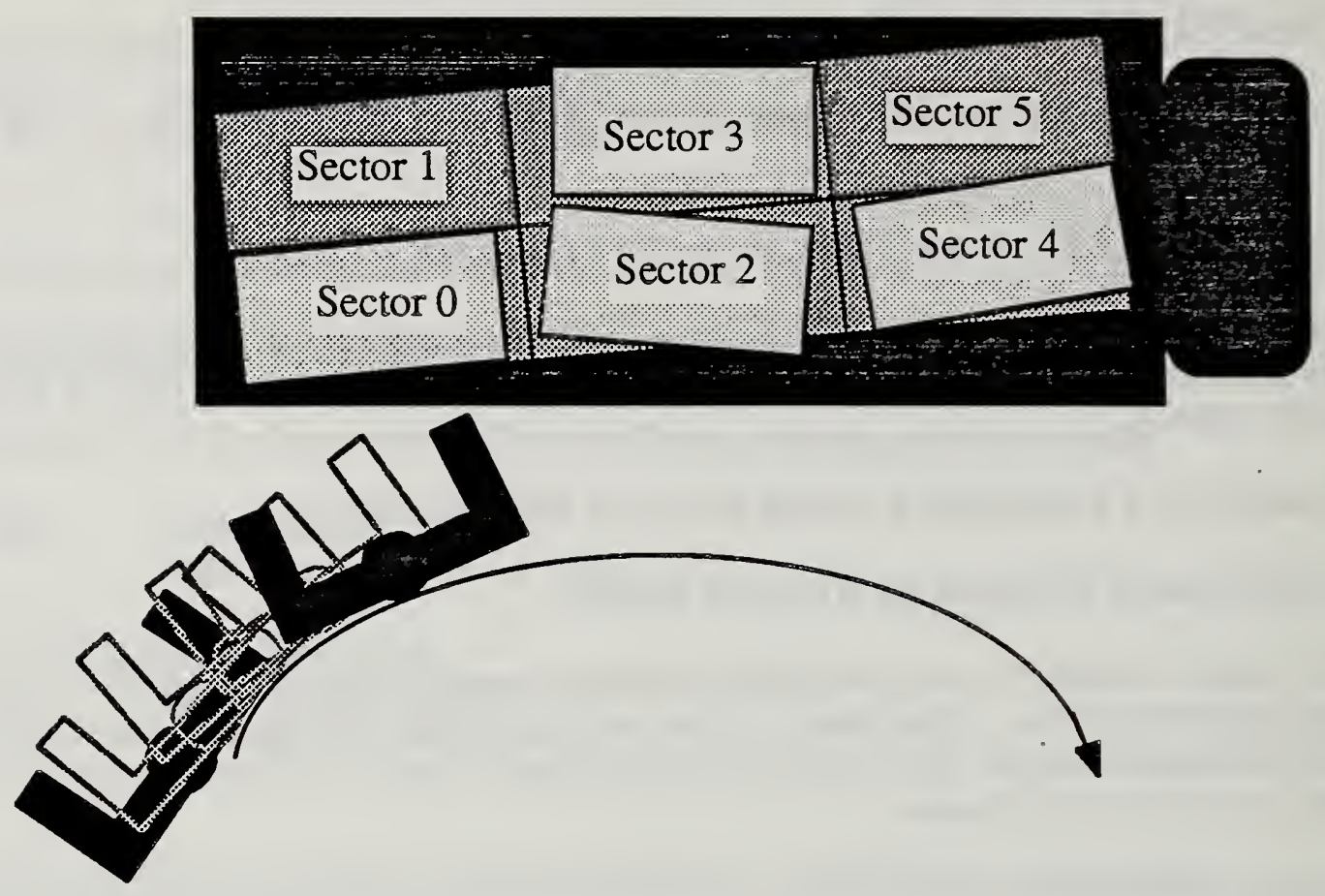

Figure 4.1. FMR searching for an array of $155 \mathrm{~mm}$ pallets randomly situated on a truck.

Because the source of the TRANSFER command is an array (section 4.1.2. addresses the syntax of the TRANSFER command) all six paths are executed for each sector of the array to be transferred. There is one exception. Since the position of the array is not known beforehand the first iteration of the TRANSFER command must include a search for the array, or in other words, the search for sector 0 is different from the search for the remaining sectors. Two differing move-to paths are used to accommodate the initial array search from the subsequent pallet search operations. The TRANSFER command reconciles which path to choose using parameters from the command and the paths. In the example plan, the move-to which describes the path from the location HOME to the array TRUCK is executed the first time to search for the array. Each remaining sector uses the move-to which describes the path from the location CONVEYOR back to the array TRUCK. Note that this path takes advantage of the array structure to execute a minimum search in acquiring the pallet.

( Scan the truck from left to right searching for the left edge of the array, align with the pallet in sector 1 , engage the fork tines, and return the location of the array) -path- move-to PALLET 1 loc HOME arr TRUCK

( Go to a predefined location where the truck bed will be using a fast joint trajectory motion.)

-ppt- goto goal nul

joint 30.030 .05 .0

(Achieve proper roll orientation.)

-ppt- equate $\begin{array}{lllll}0 & 2 & .5 & .0 & \mathrm{X} \\ \text { true }\end{array}$

tool nul

$\begin{array}{llllll}\text { cart } & .2 & .15 & .25 & .3 & .25\end{array}$ 
( Achieve proper pitch orientation.)

-ppt- equate $30.520 .0 \mathrm{Z}$ true tool nul

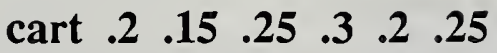

(Achieve proper elevation from truck bed.)

-ppt- range $018.00 .50 \mathrm{Y}$ true

tool nul

cart $\quad .2 \quad 15 \quad 25 \quad .3 \quad 2.25$

( Now look for the edge of the array.)

-ppt- edge 150.0 .0 false

tool SCAN-MTB

joint 25.025 .05 .0

( Guarantee sonar 1 is on the first pallet.)

-ppt- goto loc tool -CONE

joint 25.025 .01 .0

( Since two sonars, 1 and 5 , are on pallet align with pallet.)

-ppt- equate $15.50 .0 \mathrm{Y}$ true

tool nul

cart $\begin{array}{llllll}2 & .25 & .25 & .25 & .25 & .25\end{array}$

(Make sure fork is within 25 inches of pallet.)

-ppt- range $120.001 .0 \mathrm{X}$ true

tool nul

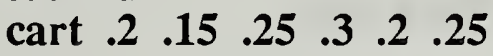

(Bring sonar 8 onto the pallet.)

-ppt- edge 828.0 .0 false

tool -TOOL

$\begin{array}{lllllllll}\text { cart } & .2 & .25 & .25 & .25 & .25\end{array}$

(Lower fork so that the lower base of the pallet acts as the sonar target.)

-ppt- range $152.00 .25 \mathrm{Y}$ true

tool nul

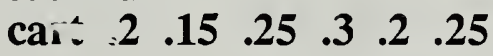

( Achieve fine alignment with pallet entry side)

-ppt- approach-pallet

7 17.75 .5

$\begin{array}{lll}15 & 28.5 & .25\end{array}$

1015.0 .25

$\begin{array}{lllll}7 & 6 & .0 & .5 & \text { true }\end{array}$

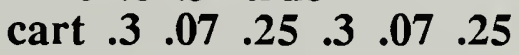

( Guide fork underneath the pallet until within range of sonar 12.)

-ppt- pickup-pallet $7 \quad 4.00 .5$ true

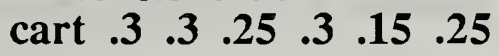

( Use sonar to achieve precise servoed distance.)

-ppt- range $123.0 .25 \mathrm{X}$ true 
tool nul

$\begin{array}{llllllll}\text { cart } & .2 & .15 & .25 & .25 & .2 & .25\end{array}$

( Move in the final distance to engage pallet.)

-ppt- goto tool PALLET-ENGAGE

cart $\begin{array}{llllllll} & .3 & .3 & .25 & .3 & .3 & .25\end{array}$

( Now return pose which defines the location of the array.)

-ppt- return-pose TRUCK

( This path is used to engage the fork with all remaining pallets in each sector of the array TRUCK. It is executed after the first pallet is transferred to the conveyor.)

-path- move-to PALLET 1 loc CONVEYOR arr TRUCK

( Move to a position in front of next sector of array. Note that the TASK level will automatically index to the next sector because 1 , the path destination is an array and 2, the path point location type is goal.) -ppt- goto goal SECTOR-SAFE joint 25.025 .05 .0

(Lower fork so that the lower base of the pallet acts as the sonar target.)

-ppto range $152.00 .25 \mathrm{Y}$ true tool nul cart $.2 \quad \begin{array}{llllllll}2 & .15 & .25 & .3 & .2 & .25\end{array}$

(Achieve fine alignment with pallet entry side)

-ppt- approach-pallet

$$
\begin{aligned}
& \begin{array}{llll}
7 & 17.75 & .5
\end{array} \\
& \begin{array}{lll}
15 & 28.5 & .25
\end{array} \\
& \begin{array}{llll}
10 & 15.0 & .25
\end{array} \\
& \begin{array}{lllll}
7 & 6 & .0 & .5 & \text { true }
\end{array}
\end{aligned}
$$

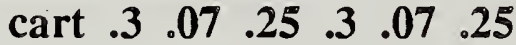

( Guide fork underneath the pallet until within range of sonar 12.)

-ppt- pickup-pallet $74.0 \quad 0.5$ true

$$
\text { cart } \begin{array}{lllllll}
3 & .3 & .25 & .3 & .25
\end{array}
$$

( Use sonar to achieve precise servoed distance.)

-ppt- range

$$
123.0 .25 \times \text { true }
$$

tool nul

$\begin{array}{llllllllllll}\text { cart } & .2 & .15 & .25 & .3 & .2 & .25\end{array}$

(Move in the final distance to engage pallet.)

-ppt- goto tool PALLET-ENGAGE

$\begin{array}{lllllllll}\text { cart } & .3 & .3 & .25 & .3 & .3 & .25\end{array}$

( The approach to the pallet is handled by the initial move-to, thus this path is empty.)

-path- approach-pickup PALLET 1 arr TRUCK

(All paths require at least one path point command, in this case a goto tool 
nul.)

-ppt- goto tool nul

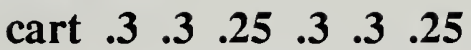

The depart-pickup, move-to, approach-release and depart-release are identical to the corresponding paths for the randomly oriented pallet in Section 4.1.1.

\subsubsection{The RSL Command to Transfer an Array of Pallets}

The command to transfer an array of pallets based on the plan described in Section 4.2.1. has the following syntax:

\section{TRANSFER PALLET 1 arr TRUCK 0243 ; loc CONVEYOR}

The command is entered from the TASK level (RCS Manual Chapter 9.4, page 9-7). The robot must currently be at the location HOME or a nul-path error will be generated (unless another path is mistakenly found). The sector list for the array TRUCK in the above command shows how individual pallets are selected for transfer and in what order. The commanded transfer sequence for the array is sectors $0,2,4$ and 3. 


\section{The FMR PATH Level}

This section serves as an augmentation to The NBS Real-time Control System User's Reference Manual section on the PATH level (section 10.5) and closely follows the format of that section.

\subsection{PATH Commands}

FMR conforms to this section.

\subsection{PATH Input Command Buffer}

FMR conforms to this section.

\subsection{PATH Status Information}

The status-arg-out values have been extended for the FMR. The status values are now centrally located in the VAR-O error-list (located in block 10 of the RSL level). This can be viewed in show mode from any level. The complete list of the status values and their meaning follow.

\section{System errors}

0 noerror

-1 programmer - Programming error. Check last piece of code compiled.

-2 command-error - invalid command.

\section{TASK errors}

101 task-cmd - invalid argument in command.

102 nul-path - path can't be found. Hasn't been loaded or defined by the user.

103 path-error - error reported by PATH level.

\section{PATH errors \\ 200 path-cmd - invalid argument in command. \\ 201 ppt-para - invalid Path Point command or parameter. \\ 202 prim-error - error reported by PRIM level.}

203 sensor-cond - robot paused because some sensor condition was not met. The PATH level variable ppt-command will show the current Path Point command which reported the error.

204 point-reached - reached goal before sensor condition met. Usually means an edge condition was not met before the goal was reached. Look at the VAR-O edge-var and edge-para to determine which edge conditions were not met. no-echo-sonar - sonar reading shows no object detected. sonar-limit - object is closer then lower limit of the selected sonar. no-pallet - the scan routine did not detect a pallet. This is normally caused by the pallet being outside the pallet-area parameter (set when the scan path point is compiled; try raising the limit) or by the pallet right edge not being detected (move pallet closer to the beginning of the scan not towards the goal destination of the scan or increase the rotation of the scan destination movetable).

208 sw-bad - indicates a faulty switch. In theory the switches cannot detect both pallet feet simultaneously. Thus, if switches on both tines are active, one switch must be faulty. There may be dirt on the lens, or worse the switches are actually seeing both pallet feet because of a misalignment of the switches, the sensitivity of a switch has increased or the pallet feet are not the expected distance apart.

209 no-sonar - a sonar has been selected but the model for the sonar has not been compiled. Check to see if the entry exists for the sonar by showing snr-model-array on the 
PATH level. A 0 in an element indicates there is no entry for the particular sonar.

\subsection{PATH Errors}

FMR conforms to this section.

\subsection{PATH Processing}

FMR conforms to this section.

\subsection{PATH Preprocessing}

The routine that reads the sonars has been added to the preprocess portion of the PATH control cycle. The sonars have several modes of operation which are described in Section 5.6.1.2. The programmer selects the desired sonar to read and waits for the range data to arrive. This process is described in section 5.6.1.

\subsubsection{SONAR-READ}

\section{Input: sonar-request}

Output: sonar-range, sonar-valid, echo-valid, low-lim

A routine that wishes to read a particular sonar(s) need only set the element of the sonar-request array corresponding to the desired sonar to true. The smacro statement to select sonar 0 is:

true $=>$ sonar-request $\{0 \#\}$

would enable the sonar. The variable sonar-valid will return the value true when the sonar's range data has been set. The data is stored in the array sonar-range and can be accessed from within a routine in the following manner (again for sonar 0 );

sonar-range $\{0 \#\} .=>$. temporary-storage

or from the terminal in show mode as such.

\section{:S sonar-range}

SONAR-READ tests status, which if equal to error, will not over-write the sonar values during the error cycle. Physically, the desired sonars are enabled by SELECT-SONAR which also sets the bits in the variable curr-sel-sonar to one for each sonar selected. If a sonar is selected, the routine READ-SONAR-DATA is called.

The following routines are associated with the sonar sensors.

\subsubsection{SELECT-SONAR}

Input: prev-sel-sonar, prev-sel-mode

Output: curr-sel-sonar, curr-sel-mode

The primary responsibility of this routine is to physically control the sonar sensors.

GET-SONAR-SELECTION is called to set curr-sel-sonar. If curr-sel-sonar is not equal to prev-sel-sonar then the user has changed the selection of the sonar. This logic is also tested against curr-sel-mode which determines in what mode the user desires the sensors to be run (see 5.6.1.2. RESET-SONAR). RESET-SONAR is called if the same sonar needs to be fired again (by having its status cleared) or if the user's sensor requirements have changed. 


\subsubsection{RESET-SONAR}

\section{Input: flood-sonar}

Output: sonar selection and mode

This routine calls the routines that write to the sensor electronics and initializes key variables. If the sonars are operating in flood fashion, that is all sonars fire continuously but the ones selected via sonar-request are the only ones read, then there is no need to call

OUTPUT-SONAR-SELECTION. In this case, the sonars are turned on once by the routine FLOOD-SONAR.

The two modes the sonars operate in are free-run (sonars are fired continuously via external clock) and one-shot (sonars are fired once via user software). The modes can run in either flood fashion where all sonars are physically fired, or non-flood fashion where only the sonars selected via sonar-request are physically fired. The sonars are typically used in a flood fashion which increases the probability that a signal from one of the sonars (not necessarily a sonar-request sonar) will reflect off a surface and be processed by a selected sonar. Note that the reading may not be exact but it will allow the robot to proceed and possibly extract more accurate information.

The mode is set using OUTPUT-SONAR-MODE. The variable sonar-on, when written to port j1-c, resets all the sonar status bits. This is needed before the next echo can be received.

\subsubsection{OUTPUT-SONAR-SELECTION}

Input: curr-sel-sonar

Output: sonar selection

Writes the low byte of curr-sel-sonar out to port j1-a and then the high byte.

\subsubsection{OUTPUT-SONAR-MODE}

Input: curr-sel-mode

Output: mode

Writes the variables which contains the code for the desired sonar mode to port j1-c.

\subsubsection{READ-SONAR-DATA}

Input: sonar-rdy-status, \#of-cycle-waiting

Output: sonar-valid, need-one-shot

This routine keeps track of when to read the sonars. The status of each sonar currently selected is set from GET-SONAR-STATUS. sonar-rdy-status has each bit set to one for the appropriate sonar having valid range data. This is compared to curr-sel-sonar (since they both keep track of the desired sonar in a bit map fashion) to find out when all the sonars are ready to be read. Some sonars may not return a ready status ever (the echo is never received). To prevent an endless loop situation, \#of-cycle-waiting keeps track of how many cycles have passed. If it exceeds \#of-cycle-allowed then the waiting stops and the sonars that have valid readings as well as the errant sonars are all set using SET-SONAR-RANGE. Finally sonar-valid is set true to indicate that the sonar values in sonar-range are now valid, and need-one-shot is set true which allows SELECT-SONAR to clear the status bit for the next range reading.

\subsubsection{GET-SONAR-STATUS}

Input: curr-sel-sonar Output: sonar-rdy-status 
The status of sonars 0 thru 7 are read in and then sonars 8 thru 15 . They are masked with curr-sel-sonar to remove status information from sonars that have not been selected by the user. Status will arrive from unwanted sonars when they are operated in a flood fashion since all sonars are fired in this case. The correct status is returned in sonar-rdy-status.

\subsubsection{SET-SONAR-RANGE}

Input: curr-sel-sonar, sonar-rdy-status, snr-model-array

Output: sonar-range

This routine sets the range values of all sonars selected by the user regardless if an echo has arrived or not. Each sonar is tested to see if it has been selected. If so the sonar model is retrieved. If a status has arrived for the sonar then the raw range data is retrieved using GET-SONAR-DATA. The raw value is then converted and stored into sonar-range. If the converted range is less then the minimum detectable range then the flag min-limit is set true. If the status bit indicates no echo from the sonar then the maximum range of the sonar, snr-max-range, is stored in sonar-range.

\subsubsection{GET-SONAR-DATA}

\section{Input: sonar-index}

Output: J2-A

This routine reads one sonar range value from the sonar interface box. sonar-index selects which sonar to read. $\mathrm{jj}$ is set according to sonar-index, a 0 if sonar-index is between 0 and 7 or a 1 if between 8 and 15. J1-B is set to select the sonar within the two groups (0-7 or 8-15). If the low group is chosen, the high byte is selected and read in, then the low byte is selected, read in and added to the high byte. If the high group is chosen, the high byte is selected and read in, then the low byte is selected, read in and added to the high byte. The raw range data is returned in $\mathbf{J} \mathbf{2}-\mathbf{A}$.

\subsubsection{CLEAR-SONAR-REQUEST}

This routine sets all elements of the sonar-request array to false, clearing any requests.

\subsubsection{SONAR-OFF}

This routine clears all sonar requests and shuts off all the sonars.

\subsection{PATH Decision Processing}

The bulk of the FMR application resides in the routines that execute the path points. These routines which are an extension to the routines listed in the RCS Manual are covered in this section.

\subsubsection{SEND-HALT}

Input: halt-enable, halt-request, prim-status

Output: ppt-done

This routine is used by other Path Point commands to halt the robot and is highly tailored for this purpose. A HALT will be executed if the halt-enable flag equals true (this is done when the path point parameter is compiled). The halt-request variable is primarily used to ensure that only one HALT is executed. HALT selects the desired halt pose. PRIM will report done when it has reached the halt pose. Since this routine is used quite often by other path point routines the flag ppt-done is set true (if the halt pose is reached while the sensor condition is still met) at the termination of this routine. 


\subsubsection{HALT}

Input: tool-pose-^, traj-type

Output: prim-com-pose, traj-para

This routine commands PRIM to halt at the current position as defined by the pose stored in prim-com-pose. The halt pose is dependent on the commanded trajectory since PRIM will place the tool pose at the commanded PRIM pose for a cartesian trajectory and will place the sensor at the commanded PRIM pose for a servo trajectory.

The current pose of the tool frame is first retrieved. If the trajectory type is servo then the sensor movetable is added to the tool pose and stored in prim-com-pose, else the tool pose is stored in prim-com-pose.

\subsubsection{TRANSLATE}

Input: tool-pose-^, delta, axis

Output: prim-com-pose

This routine constructs a pose in prim-com-pose that is a translation of delta along the tool frame axis.

\subsubsection{ROTATE}

Input: tool-pose- $\wedge$, delta, axis

Output: prim-com-pose

This routine constructs a pose in prim-com-pose which is a rotation of delta about the pallet frame axis. An attempt has been made to modify the rotation such that the positional relationship
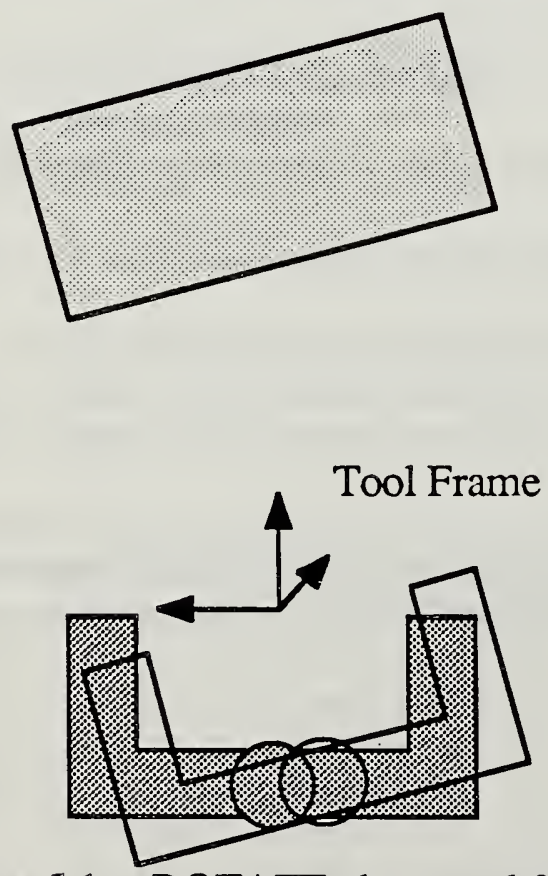

Figure 5.1a. ROTATE about tool frame

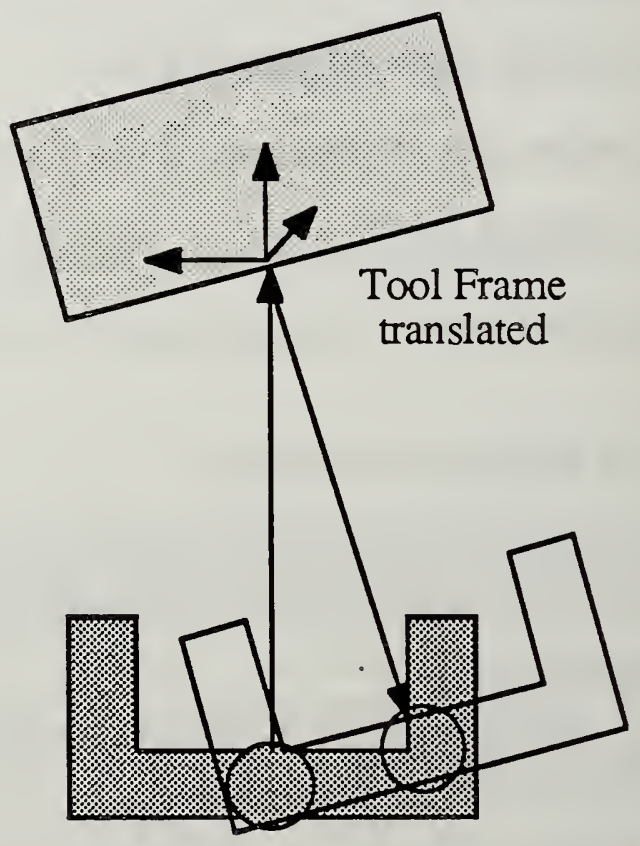

5.1b. ROTATE about tranislated tool fame

between the pallet and the fork does not change. Figure 5.1a shows a rotation motion about the tool frame. Figure $5.1 \mathrm{~b}$ shows a more desirable motion taking place about the pallet frame by preceding the rotation by a translation (derived from sensor range data). This maintains the proper relationship between the fork mounted sensors and the pallet. 


\subsubsection{INIT-SONAR-MODEL-ARRAY Input: SONAR-MODEL-FILE \\ Output: snr-model-array}

This routine retrieves the record numbers of the sonar model entries in the file

SONAR-MODEL-FILE and stores them in the array snr-model-array. The sonar model variable snr-id is used to index into the sonar-model-array such that the pointer to the sonar model record for sonar number 1 is found in element 1 of snr-model-array. For information on the sonar model itself see section 2.1. Sonar Modelling.

\subsubsection{Range Path Point Routines}

The following routines are associated with the RANGE Path Point command (section 3.1.).

\subsubsection{RANGE-PPT}

Input: new-ppt

Output: RANGE routines

This is the high level routine called during the execution of the RANGE Path Point command.

The first time executed it initializes all variables, all remaining executions call RANGE.

\subsubsection{RANGE-PPT-INIT}

Input: RANGE-FILE

Output: range-para, TRAJ-PHRASE, halt-enable, halt-request, sonar-request

This routine retrieves the parameters compiled in the Path. It uses these parameters to turn on the needed sonars and to retrieve the trajectory parameters.

\subsubsection{RANGE-SONAR-MODEL-INIT}

Input: snr-model-array

Output: snr-model-para

This routine retrieves the sonar model for the chosen range sonar.

5.7.6.4. RANGE

Input: sonar-valid, min-limit, range-para

Output: delta, axis, halt-request, TRANSLATE, RANGE-GOAL, PRIM-TRAJ,

SEND-HALT

This routine generates an error value, delta, as the difference (in inches) between the sonar range reading and the desired range reading ( $r$-range).. If min-limit equals true then this routine will not function properly and therefore reports an error. This can happen, for example, if a Polaroid sensor is chosen instead of a Migatron sensor to range to a value of 2 inches. If the sensor is at 5 inches, a forward motion is commanded. But since the value does not change (because the Polaroid sensor will not operate at 5 inches) this routine continues to command forward motion.

If delta is less then $\mathbf{r}$-thresh then SEND-HALT is called to bring the robot to a halt. If delta exceeds r-thresh then depending on the trajectory type RANGE-GOAL or TRANSLATE is called to calculate a new goal pose which will minimize the difference between the desired range and the actual range. Because the robot can overshoot the goal which will cause the sonar readings to again generate an error value greater then $\mathbf{r}$-thresh, this routine may be called several times. In this case halt-request is set false which clears any previous calls to SEND-HALT. 


\subsubsection{RANGE-GOAL}

Input: tool-pose-^, snr-mtb-^, axis, delta

Output: prim-com-pose

This routine constructs a pose in prim-com-pose that is a translation of delta along the sonar frame axis. It is used to position the sonar at the goal pose as opposed to TRANSLATE which positions the tool frame at the goal pose.

\subsubsection{EDGE Path Point Routines}

The following routines are associated with the EDGE Path Point command (section 3.2.).

\subsubsection{EDGE-PPT}

Input: new-ppt

Output: EDGE routines

This is the high level routine called during the execution of the EDGE Path Point command. The first time executed it initializes all variables, all remaining executions call EDGE.

\subsubsection{EDGE-PPT-INIT}

Input: EDGE-FILE

Output: edge-para, TRAJ-PHRASE, LOC-PHRASE, halt-enable, halt-request, sonar-request

This routine retrieves the parameters compiled in the Path. It uses these parameters to turn on the needed sonars and to retrieve the trajectory and location parameters.

\subsubsection{EDGE-SONAR-MODEL-INIT}

Input: snr-model-array

Output: snr-model-para

This routine retrieves the sonar model for the chosen edge sonar.

\subsubsection{EDGE}

Input: sonar-valid, range-set, range-para

Output: current-range, initial-range, ?EDGE, SEND-HALT

This routine tests for the conditions that satisfy a perceived edge. The first valid sonar reading is saved in initial-range. This is required to satisfy the first edge condition that a change in range between the first reading and the current reading must exceed e-delta. Once the value is saved PRIM-TRAJ is called to start the robot moving to the goal pose (a parameter of EDGE). The second edge condition requires that the range values, starting with initial-range and ending at the current-range, must cross the value given in the parameter e-range. This allows the user to specify at what range to look for the edge. Figure 5.2 shows an example of an edge observed at a range of 50 inches. This edge was distinguished from the other edges by selecting an e-delta of 30 inches and an e-range of 60 inches. Both conditions are tested and if they are true SEND-HALT is called to bring the robot to a halt. If they are not true then ?EDGE is called to determine if an error has occurred. 


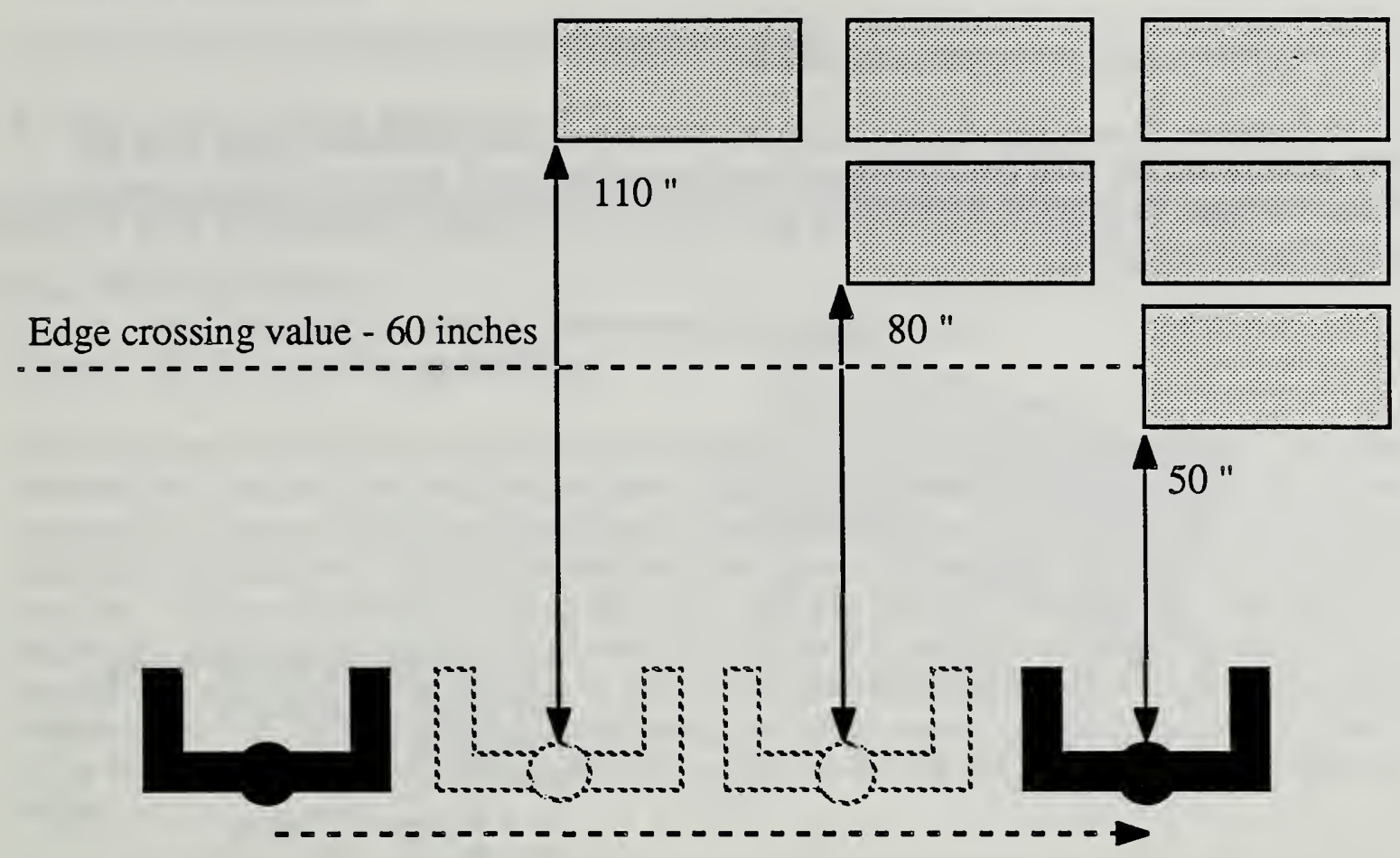

Figure 5.2. An edge threshold of 60 inches "filters" the edge from 110 inches to 80 inches but passes the edge from 80 inches to 50 inches during the sensor scan.

\subsubsection{5. ?EDGE}

Input: prim-status, halt-request

Output: PRIM-PAUSE, status, status-arg

This routine tests the status from the PRIM level to determine if the robot has reached the goal pose without the edge conditions being satisfied. If this occurs the error sensor-cond is returned in status-arg.

\subsubsection{EQUATE Path Point Routines}

The following routines are associated with the EQUATE Path Point command (section 3.3.).

\subsubsection{EQUATE-PPT}

Input: new-ppt

Output: EQUATE routines

This is the high level routine called during the execution of the EQUATE Path Point command. The first time executed it initializes all variables, all remaining executions call EQUATE.

\subsubsection{EQUATE-PPT-INIT}

Input: EQUATE-FILE

Output: equate-para, axis, TRAJ-PHRASE, halt-enable, halt-request, sonar-request

This routine retrieves the parameters compiled in the Path. It uses these parameters to turn on the needed sonars, set the axis of rotation and retrieve the trajectory parameters.

\subsubsection{EQUATE-SONAR-MODEL-INIT}

Input: snr-model-array, snr-model-para, e-axis 


\section{Output: snr-model-para, snr-sprtn, snr-offst}

This routine retrieves the models for both sonars. The data from the model along with the EQUATE parameters are used to determine the geometry required for rotation using the range data from two sonars. Figure 5.3 shows the geometry for two sonar rotation (the axis of rotation is perpendicular to the page).

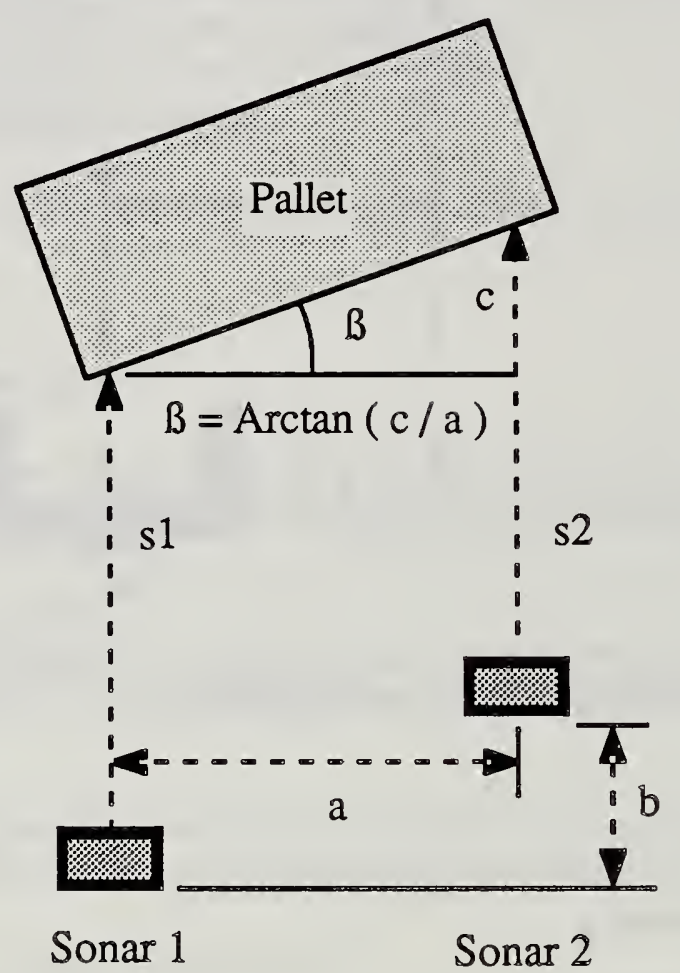

$B=$ Rotation angle for equate

$\mathrm{a}=$ Sonar separation

$\mathrm{b}=$ Sonar offset

$\mathrm{c}=\mathrm{s} 1-\mathrm{s} 2+\mathrm{b}$

s1 = Range of Sonar 1

s2 = Range of Sonar 2

Figure 5.3. Geometry for EQUATE rotation.

After the sonar models have been retrieved, the position of each sonar is extracted and stored in the vectors e1-xyz and e2-xyz. The vectors are to extract the rotation parameters: sonar separation (snr-sprtn) and sonar offset (snr-offst).

\subsubsection{EQUATE}

Input: sonar-valid, equate-para

Output: SEND-HALT, NOT-EQUATED

This routine generates an error value, eq-delta, as the difference (in inches) between the sonar readings. If eq-delta is less then e-threshold then SEND-HALT is called to bring the robot to a halt. If eq-delta exceeds e-threshold then NOT-EQUATED is called to calculate a new goal pose which will minimize the difference between the sonars.

\subsubsection{NOT-EQUATED}

Input: eq-delta, snr-sprtn

Output: delta, ROTATE, PRIM-TRAJ

This routine calculates the angle delta which is the arctangent of the quotient eq-delta and snr-sprtn. The delta is clipped to within plus or minus eq-max. Because the robot can overshoot the goal which will cause the sonar readings to again generate an error value greater then e-threshold, this routine may be called several times. Thus, halt-request is set false 
which clears any previous calls to SEND-HALT.

\subsubsection{SCAN Path Point Routines}

The following routines are associated with the SCAN Path Point Command (section 3.4.).

\subsubsection{SCAN-SONAR}

Input: new-ppt, prim-status, more-records, right-edge-^

Output: SCAN routines, ppt-done

This routine commands the robot to search the work volume for a pallet load. The search path is selected by the user but the direction must be from left to right (although this can be easily changed). Sonar readings and positions of the robot during the read are stored for analysis. The first time the routine is called all necessary variables are initialized and the robot is started in motion. The scan terminates when the robot reaches the goal or when the right edge of the pallet is found. If the right edge is not found and the goal is reached a no-pallet error is returned in status-arg (pallet may be to close to the start or the end of the search path). The pointer right-edge- $\wedge$ is set to the record containing the range value and position of the robot when the edge was observed. It remains a 0\# (nul) until the SAVE-SCAN-ROUTINE detects the right edge.

Once the right edge has been detected RETURN-SCAN-POSE is called to write out the pose indicating the closest feature of the pallet to the robot. N1-N2-SET is called to return values needed by the ALIGN-GRIP Path Point command.

\subsubsection{SCAN-INIT}

Input: SCAN-FILE

Output: scan-para, LOC-PHRASE, TRAJ-PHRASE, halt-enable, sonar-request, pallet-min-range, SONAR-FILE-INIT, PRIM-TRAJ

This routine retrieves the parameters compiled in the Path. It uses these parameters to turn on the selected sonar and to retrieve the trajectory and location parameters. SCAN-VAR-INIT is called to initialize pertinent variables. SONAR-FILE-INIT initializes the file that stores the scan readings.

\subsubsection{SCAN-SONAR-MODEL-INIT \\ Input: snr-model-array \\ Output: snr-model-para}

This routine retrieves the sonar model for the chosen scan sonar.

\subsubsection{SAVE-SCAN-READING}

Input: sonar-valid, skip-reading, skip-till

Output: skip-reading, ADD-TO-SONAR-REC

This routines saves the scan reading by calling ADD-TO-SONAR-REC to store the sonar reading and actual robot pose to file SONAR-REC. Variable skip-till allows valid sonar readings to be skipped to conserve memory.

\subsubsection{ADD-TO-SONAR-REC}

Input: left-edge- $\wedge$, right-edge- $\wedge$, ass/rec, $\max /$ rec

Output: ADD-RECORD, SET-PALLET-MIN-RANGE, LEFT-EDGE-TEST, RIGHT-EDGE-TEST 
This routine is responsible for storing the scan data and determining an estimate of the position of the pallet. ADD-RECORD stores the sonar range value and the pose of the robot into the file SONAR-FILE. SET-PALLET-MIN-RANGE ensures that the minimum range reading during the scan is stored in pallet-min-range and temp-min-pose- ${ }^{\wedge}$ points to the record that contains the scan reading. LEFT-EDGE-TEST tests for the left edge of the pallet and returns the number of the scan record associated with the edge in left-edge ${ }^{\wedge}$.

RIGHT-EDGE-TEST performs the same function for the pallet right edge, the scan record is returned in right-edge- $\wedge$.

\subsubsection{ADD-RECORD}

Input: sonar-range, tool-pose-^, s-ptr

Output: save-sonar-var, save-range

This routine stores the sonar range and the tool pose into the save-sonar-var record of file SONAR-FILE. The pointer s-ptr keeps track of the next available record in the file. This prevents the routine add-record from searching the file for a new record. Each record includes a field called back-link which is used to link the file backwards. This assists in file manipulations in later routines.

\subsubsection{SET-PALLET-MIN-RANGE}

Input: save-range, pallet-min-range, s-ptr

Output: pallet-min-range, temp-min-pose

This routine ensures that pallet-min-range contains the minimum range reading during the scan and that temp-min-pose- ${ }^{\wedge}$ points to the associated SONAR-FILE record. The initial value of pallet-min-range is initialized to the parameter pallet-area in SCAN-INIT.

\subsubsection{LEFT-EDGE-TEST}

Input: save-range, pallet-area, left-edge- $\wedge$, l-e-cnt, l-e-thresh, s-ptr

Output: l-e-cnt, left-edge-^^, LEFT-EDGE-ADJUST

This routine tests for the left edge of the pallet. A sonar range less then pallet-area indicates the possibility of the pallet edge. To filter out any noise typically generated from the sonar side lobes several valid reading are required before the edge is accepted. A count of the edge readings is kept in l-e-cnt. If the count exceeds the threshold in l-e-thresh then left-edge- $\wedge$ is set to the current record associated with the edge reading. Since the reading is actually the last in a sequence of valid edge readings LEFT-EDGE-ADJUST is called to reset left-edge-^ to the first valid reading. l-e-cnt is reset to $0 \#$ whenever a value greater then pallet-area is encountered to ensure that the sequence of edge values are all valid (ie. one false reading starts the edge filtering algorithm over).

\subsubsection{LEFT-EDGE-ADJUST}

\section{Input: left-edge-^, l-e-cnt, pallet-area}

Output: left-edge-^ $\wedge$, d01

This routine resets left-edge-^ to the first valid reading of the pallet left edge. Left-edge- $\wedge$ initially contains the last reading of the edge and is used to start the backwards search in SONAR-FILE. The search terminates when a range reading greater then pallet-area is encountered. The record which has the first valid edge reading is stored into left-edge- $\wedge$. The range value of the edge is stored into $\mathrm{d} 0 \mathrm{l}$. The coordinates of the edge can be obtained by transforming the pose in the field scan-pose a distance of $\mathrm{d} 0 \mathrm{l}$ in the plus $\mathrm{X}$ direction.

\subsubsection{RIGHT-EDGE-TEST}


Input: save-range, pallet-area, left-edge-^, right-edge-^, r-e-cnt, r-e-thresh, s-ptr

Output: r-e-cnt, left-edge-^, RIGHT-EDGE-ADJUST

This routine tests for the right edge of the pallet. It operates in a manner similar to

LEFT-EDGE-TEST except it test for range values greater then pallet-area.

\subsubsection{RIGHT-EDGE-ADJUST}

Input: right-edge-^, r-e-cnt, pallet-area

Output: right-edge- $\wedge, \mathrm{d} 0 \mathrm{r}$

This routine resets right-edge- $\wedge$ to the last valid reading of the pallet left edge. It operates in a manner similar to LEFT-EDGE-ADJUST except the terminating condition of the edge search is a range value less then pallet-area.

\subsubsection{RETURN-SCAN-POSE}

Input: temp-min-pose-^

\section{Output: FIND-LEFT-CORNER, FIND-RIGHT-CORNER, SET-RETURN-POSE}

This routine is responsible for refining the position of the pallet and returning the pallet pose.

The pallet pose is derived from the scan information. The scan routine was not intended to be the final routine used for determining the entry side of the pallet. It has not been investigated to see if this determination can be made in all cases. Therefore the pallet pose is the pose of the scan where the closest feature of the pallet was observed plus a few adjustments.

One adjustment is made to accommodate the three types of possible orientations that can exist between the pallet and the robot; tilt, long side and short side. The closest feature of the pallet when it is tilted (a corner) can be accurately derived but for the long and short side cases it is desirable to attain the center of the pallet side. Since the pose defining the side may not be the center an algorithm is used to derive the center. Figure 5.4a-c shows three examples of pallet orientation with all the features labeled. The short side case looks similar to the long side case shown in Figure $5.4 \mathrm{~b}-\mathrm{c}$ and therefore is not shown. The pose pointed to by min-pose- ${ }^{\wedge}$ is the actual pallet pose returned.

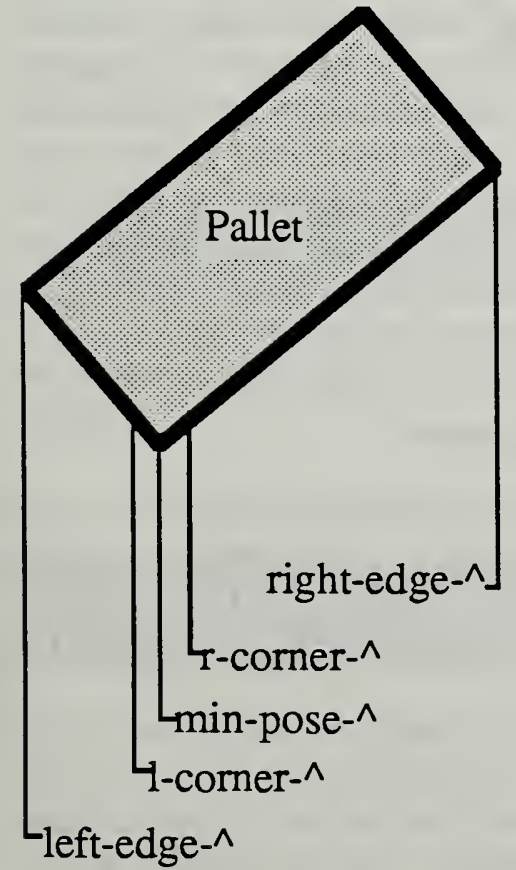

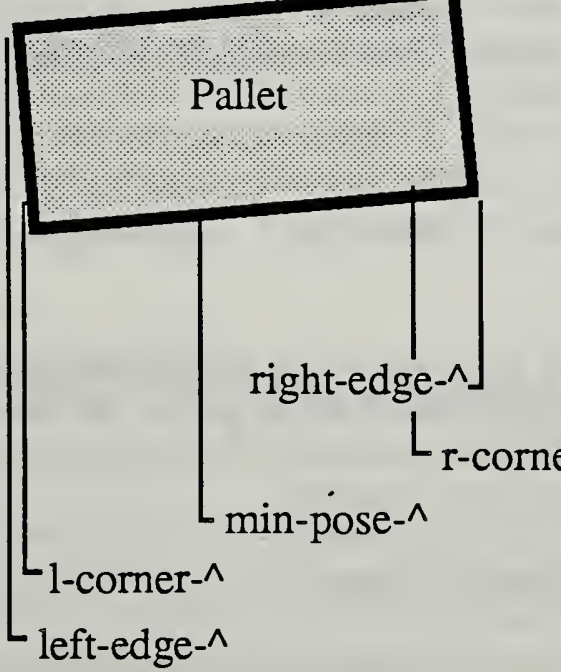

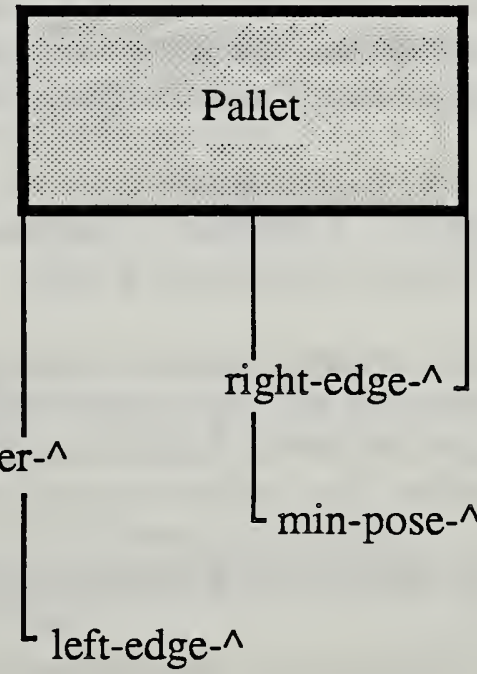


Figure 5.4a. Pallet Tilt Case.

4b. Pallet Long Case with slight tilt. 4c. Pallet Long Case with no tilt.

The first step of the center detection algorithm is to group together a set of scan values that are within a user selectable threshold (closest-point-delta) of the closest range observed. The end points of the group are stored in $\mathbf{r}$-corner ${ }^{\wedge} \wedge$ and $\mathbf{l}$-corner ${ }^{\wedge} \wedge$ (Figure 5.4a-c). The routines FIND-LEFT-CORNER-EDGE and FIND-RIGHT-CORNER-EDGE calculate these values.

The only remaining step in the algorithm is to find the middle entry in the group of scan values. The routine SET-RETURN-POSE locates the middle entry (min-pose- ${ }^{\wedge}$ ) and retrieves the scan pose. The scan pose is translated out along the $\mathrm{X}$ axis of the tool frame a distance specified by the parameter pallet-min-range and then written out to the pose record pointed to by the parameter scan-return-pose-^.

\subsubsection{FIND-LEFT-CORNER-EDGE}

Input: temp-min-pose- $\wedge$, pallet-min-range, save-sonar-var, scan-para

Output: l-corner-^ $\wedge$

This routine searches SONAR-FILE from temp-min-pose-^ (points to record containing pallet-min-range) backwards finding the last pallet reading that is within the parameter closest-point-delta of pallet-min-range. This left edge of the closest point is pointed to by l-corner ${ }^{\wedge}$.

\subsubsection{FIND-RIGHT-CORNER-EDGE}

Input: temp-min-pose- $\wedge$, pallet-min-range, save-sonar-var, scan-para

Output: r-corner-^

This routine searches forward from temp-min-pose-^ using the same scheme as

FIND-LEFT-CORNER-EDGE to set $r$-corner-^ ${ }^{\wedge}$ to the right edge of closest point.

\subsubsection{SET-RETURN-POSE}

Input: r-corner-^, l-corner-^, bytes/record, pallet-min-range, scan-return-pose- $\wedge$

Output: min-pose-^

This routine bisects the group of records to isolate the center of the closest pallet feature. min-pose-^ points to this record. The pose is also written out to the pose pointed to by the parameter scan-return-pose-^ .

\subsubsection{N1-N2-SET}

Input: $\quad r-$ corner $^{\wedge} \wedge$, l-corner-^, left-edge- ${ }^{\wedge}$, right-edge- ${ }^{\wedge}$, min-pose-^

Output: n1, n2, n3, n1+n2

This routine performs further analysis of the pallet scan. $\mathbf{n} \mathbf{1}$ equals the number of readings taken from the left edge to the closest feature of the pallet, $\mathbf{n} \mathbf{2}$ the number of readings from the right edge to closest feature and $\mathbf{n} \mathbf{3}$ is the number of readings associated with the closest feature of the pallet. The values are used in later routines.

\subsubsection{ALIGN-GRIP Path Point Routines}

The following routines are associated with the ALIGN-GRIP Path Point command (section 3.5.). 


\subsubsection{ALIGN-GRIP}

Input: new-ppt, l-done-flag, test-called, sonar-valid

Output: GRIP-RETRIEVE, TURN-ON-SONAR, TEST-FOR-ALIGNMENT, FIND-GRIP-SIDE-STATE, ppt-done

This is the high level routine called during the execution of the ALIGN-GRIP Path Point command. The first time executed it initializes all variables and turns on the required sonars. The terminating condition for this routine is when l-done-flag equals true which means the fork is aligned with the long side of the pallet. TEST-FOR-ALIGNMENT is called one time to estimate the initial orientation of the pallet. The remaining executions wait for valid sonar data and then call FIND-GRIP-STATE to determine if a robot motion is required to align the fork with the pallet.

\subsubsection{GRIP-RETRIEVE}

\section{Input: ALIGN-GRIP-FILE}

Output: align-grip-para, halt-enable, halt-request

This routine retrieves the parameters compiled in the Path.

\subsubsection{TURN-ON-SONAR}

\section{Input:}

Output: sonar-request

This routine turns on sonars 1,5 and 8 .

\subsubsection{TEST-FOR-ALIGNMENT}

Input: $\mathrm{n} 1, \mathrm{n} 2, \mathrm{~d} 01, \mathrm{~d} 0 \mathrm{r}$, pallet-min-range

Output: align-grip-para, s-done-flag, t-done-flag, l-done-flag, l-flag, s-flag, t-flag, step-state, first-time, test-called

This routine is called one time to calculate the nominal orientation of the pallet. The pallet orientation which describes what the robot saw during the most recent scan falls into three categories; short side, long side and tilted. In the short and long side cases the closest pallet feature (pallet-min-pose) is the center of the side facing the robot; in the tilt case it is the corner of the pallet facing the robot. $\mathbf{n} 1$ and $\mathbf{n} 2$ (the number of sonar readings between the edges and the closest point) is used to separate the tilt case from the short/long side cases. If the difference between $\mathbf{n} \mathbf{1}$ and $\mathbf{n} \mathbf{2}$ is greater then the parameter tilt-ratio then the tilt case exists. To distinguish between the short and long side cases the distance between the closest pallet feature (pallet-min-range) is subtracted from the maximum range reading between $\mathbf{d 0 r}$ and $\mathbf{d} 0 \mathbf{1}$ (distance to edges) which effectively calculates the side of the pallet perpendicular to the robot. This is then compared to the parameter min-short-side. If the side is larger than min-short-side then the short side case exists, else the long side of the pallet is facing the robot.

\subsubsection{FIND-GRIP-SIDE-STATE}

Input: s-done-flag, t-done-flag, l-done-flag Output: SHORT-SIDE-PROCESSING, TILT-PROCESSING, LONG-SIDE-PROCESSING

This routine uses l-done-flag, s-done-flag and t-done-flag to call the three processing routines. The processing routines are set up as a sequence of steps which read sonars, make decisions and move the robot. SHORT-SIDE-PROCESSING and

TILT-SIDE-PROCESSING move the robot to the long side of the pallet. 
LONG-SIDE-PROCESSING then aligns the robot with the pallet.

\subsubsection{TILT-PROCESSING}

Input: $\mathbf{n} 1, \mathbf{n} 2$, step-state, ppt-done

Output: SIDE-MOVE, SIDE-EQUATE, sonar-request, ppt-done, t-done-flag, t-flag

This routine assumes that sonar 1 (the center of the fork) is facing the closest corner of the pallet and the angle of the pallet is such that the larger of $\mathbf{n} \mathbf{1}$ and $\mathbf{n} 2$ represents the long side. $\mathbf{0}$-step tests $\mathbf{n} \mathbf{1}$ and $\mathbf{n} \mathbf{2}$ and sets the +zdirection true if the long side is in the positive $Z$ direction of the tool frame and false otherwise. The parameters are set for the proceeding steps. 1-step moves to clear the corner so that SIDE-EQUATE in step 2 will be facing a side. 2 -step then equates with the long side. Now the robot is in a position to execute LONG-SIDE-PROCESSING.

\subsubsection{SHORT-SIDE-PROCESSING}

Input: d0l, d0r, step-state, sonar-range, align-grip-para

Output: SIDE-MOVE, SIDE-EQUATE, sonar-request, ppt-done, s-done-flag, s-flag

This routine assumes that sonar 1 is centered on the short side of the pallet. The greater range reading between $\mathbf{d} 0 \mathrm{l}$ and $\mathbf{d} 0 \mathrm{r}$ is assumed to be directly related to the long side of the pallet. 0-step tests this and set the parameters and +zdirection. 1-step takes sonar 1 to the edge (corner) adjacent to the chosen long side. When done it sets movtab- ${ }^{\wedge}$ to the necessary movetable needed to rotate the fork around so that it is roughly parallel to the long side. A larger rotation movetable is used if the process flow has come from LONG-SIDE-PROCESSING because it has mistaken the short side as a long side. Experience has shown this occurs most often when the side is nearly parallel to the fork and thus the need for a larger rotation. A SIDE-EQUATE had been used initially to rotate about the corner but it's execution was not reliable. 2-step executes the rotation move. 3-step then aligns the fork with the long side. Now the robot is in a position to execute LONG-SIDE-PROCESSING.

\subsubsection{LONG-SIDE-PROCESSING}

Input: d01, d0r, step-state, sonar-range, align-grip-para

Output: SIDE-EDGE, SIDE-EQUATE, sonar-request, ppt-done, l-done-flag, l-flag

This routine accomplishes the final alignment with the long side of the pallet. It has been extended to handle the error case of a short side being mistaken for a long side. step- 0 starts by determining where the processing flow has come from. If it came directly from

TEST-FOR-ALIGNMENT as a long side case it tests the sonars against the parameter perm-equal-val to determine if any alignment is necessary. If one of the sonars is off the pallet then further alignment is needed. Also since it is possible that a short side case could exist a move is set up to align the outer sonars with what should be the long side edge of the pallet. If after aligning one sonar on the pallet edge the other sonar is off the pallet then the short side case actually exists. If processing came from tilt or short case then it is not necessary to test for this error (1-step is not called).

1-step forces the previously selected sonar off the pallet. When done it sets the parameters needed to bring the sonar back on to the pallet.

2-step uses SIDE-EDGE to bring the sonar on to the pallet. Further alignment is not required if the tilt or short case existed. If long side exists then the possibility of a short case error needs to be tested by turning on the necessary sonars (which takes an additional cycle) and calling 3-step. 
3-step tests the differences between sonars 1 and 2 against parameter max-short-side. If the difference is greater (a sonar is off the pallet) then a short side pallet case exists and

SHORT-SIDE-PROCESSING must be executed, else the fork is aligned with the long side and processing is done.

\subsubsection{SIDE-EQUATE}

Input: first-time

Output: EQUATE routines

This routine is similar in execution to the EQUATE Path Point command. The first time executed it initializes all variables, all remaining executions call EQUATE.

\subsubsection{SIDE-EQUATE-INIT}

\section{Input: align-grip-para}

Output: e-threshold, e-axis, axis, TRAJ-PHRASE

This routine initializes the parameters needed for the EQUATE routine.

\subsubsection{SIDE-EDGE}

\section{Input: first-time}

Output: EDGE routines

This routine is similar in execution to the EDGE Path Point command. The first time executed it initializes all variables, all remaining executions call EDGE.

\subsubsection{SIDE-EDGE-INIT}

Input: align-grip-para, align-mtb-^

Output: e-delta, range-set, halt-request, LOC-PHRASE, TRAJ-PHRASE

This routine initializes the parameters needed for the EDGE routine.

\subsubsection{SIDE-MOVE}

Input: first-time

Output: SIDE-MOVE-INIT, ppt-done

This routine is similar in execution to the GOTO Path Point command. The first time executed it initializes all variables. It then waits for PRIM to report done.

\subsubsection{SIDE-MOVE-INIT}

Input: align-grip-para, align-mtb- $\wedge$

Output: e-delta, range-set, halt-request, LOC-PHRASE, TRAJ-PHRASE

This routine initializes the parameters and calls PRIM-TRAJ.

\subsubsection{4. -Z-DIRECTION}

Input:

Output: e-1sonar\#, e-2sonar\#, e-sonar, sonar-request, align-mtb-^, +zdirection

This routine sets up the SIDE-EDGE move for motion in the minus $\mathrm{Z}$ direction in step 1 of SHORT-SIDE-PROCESSING.

\subsubsection{5. +Z-DIRECTION}

Input: 
Output: e-1sonar\#, e-2sonar\#, e-sonar, sonar-request, align-mtb-^ , +zdirection

This routine sets up the SIDE-EDGE move for motion in the plus $Z$ direction in step 1 of SHORT-SIDE-PROCESSING.

\subsubsection{APPROACH-PALLET Path Point Routines}

The following routines are associated with the APPROACH-PALLET Path Point command (section 3.6.).

\subsubsection{APPROACH-PALLET}

Input: new-ppt, sonar-valid, $y$-r-done, $x$-r-done, $y$-t-done, $x$-t-done

Output: APPROACH-PALLET routines, SEND-HALT, PRIM-TRAJ

This is the high level routine called during the execution of the APPROACH-PALLET Path Point command. The first time executed it initializes all variables. The remaining executions wait for valid sonar data to determine if a robot motion is required to correct the fork position.

Four degrees of freedom are tested to determine if the fork is in the proper position and orientation with respect to the pallet. CALC-Y-ROT and CALC-X-ROT determine if there are deviations in orientation about the $\mathrm{Y}$ and $\mathrm{X}$ axes respectively (see Figure 3.1). With the addition of the Migatron sensors a test for orientation about the $Z$ axis can also be incorporated. Care must be taken though that the sensors will always have a target to view (ie. a sensor isn't looking off the edge of the truck bed for instance). Position deviation along the $\mathrm{Y}$ and $\mathrm{X}$ axes are handled by CALC-Y-TRANS and CALC-X-TRANS. If the status from these routines report done then SEND-HALT is called. If any of the routines report not done then PRIM-TRAJ is called to move the robot.

\subsubsection{APPROACH-PALLET-INIT}

Input: APPROACH-PALLET-FILE

Output: approach-pallet-para, TRAJ-PHRASE, halt-enable, halt-request, sonar-request

This routine retrieves the parameters compiled in the Path. It uses these parameters to turn on the needed sonars and to retrieve the trajectory parameters.

\subsubsection{APPROACH-PALLET-S-M-INIT}

\section{Input: snr-model-array}

Output: snr-model-para, x-r-sprtn, x-r-offst, y-r-sprtn, y-r-offst, y-t-offst, $x$-t-offst

This routine retrieves the sonar model for the chosen sonars. Note that the routines EQUATE-SONAR-MODEL-INIT and RANGE-SONAR-MODEL-INIT are used since APPROACH-PALLET basically duplicates the EQUATE and RANGE commands.

\subsubsection{CALC-Y-ROT}

Input: approach-pallet-para, sonar-range, $y$-r-offst, $y$-r-sprtn, prim-com-pose Output: y-r-done, prim-com-pose

This routine sets $\mathbf{y}-\mathbf{r}$-done true if the difference between the two selected sonars is less then the parameter $\mathbf{y}-\mathbf{r}$-thresh. If the difference is greater, the correction rotation is calculated as the arctangent of the quotient delta and $\mathbf{y}-\mathbf{r}-\mathbf{s p r t n}$. The correction is applied as a rotation to prim-com-pose. Note that the routine terminates after it is successful once. This will sacrifice accuracy for speed. 


\subsubsection{CALC-X-ROT}

Input: approach-pallet-para, sonar-range, $x$-r-offst, $x$-r-sprtn, prim-com-pose Output: $x$-r-done, prim-com-pose

This routine sets $\mathbf{x}-\mathbf{r}$-done true if the difference between the two selected sonars is less then the parameter $\mathbf{X}$-r-thresh. If the difference is greater, the correction rotation is calculated as the arctangent of the quotient delta and $\mathbf{x}$-r-sprtn. The correction is applied as a rotation to prim-com-pose. Note that the routine terminates after it is successful once. This will sacrifice accuracy for speed.

\subsubsection{CALC-Y-TRANS}

Input: approach-pallet-para, sonar-range, y-t-offst, prim-com-pose Output: y-t-done, prim-com-pose

This routine sets $y$-t-done true if the difference between the actual sonar range and the desired sonar range is less then the parameter $\mathbf{y}-t$-thresh. If the difference is greater, the correction translation is calculated as the difference between the two ranges. The correction is applied as a translation to prim-com-pose. Note that the routine terminates after it is successful once. This will sacrifice accuracy for speed.

\subsubsection{CALC-X-TRANS}

Input: approach-pallet-para, sonar-range, $x$-t-offst, prim-com-pose

Output: x-t-done, prim-com-pose

This routine sets $\mathbf{x}$-t-done true if the difference between the actual sonar range and the desired sonar range is less then the parameter $\mathbf{x}-\mathbf{t}$-thresh. If the difference is greater, the correction translation is calculated as the difference between the two ranges. The correction is applied as a translation to prim-com-pose. Note that the routine terminates after it is successful once. This will sacrifice accuracy for speed.

\subsubsection{PICKUP-PALLET Path Point Routines}

The following routines are associated with the PICKUP-PALLET Path Point command (section 3.7.).

\subsubsection{PICKUP-PALLET}

Input: new-ppt, range-set, switches, z-correcting, step-state

Output: PICKUP-PALLET routines, step-state

This is the high level routine called during the execution of the PICKUP-PALLET Path Point command. The first time executed it initializes all variables and retrieves the sonar model. All remaining executions guide the robot fork beneath the pallet using a sonar to initially determine the distance to travel and the proximity sensors to determine if any corrective translations are required. The algorithm to insert the tines requires that a goal pose is calculated first. If a switch indicates an obstruction (closed) then the fork is moved sideways until the switch is clear (open). After the switch is clear an additional sideways motion can be user specified to accommodate variations in the sensitivity and orientation of the proximity sensors. Before the fork is moved sideways though the distance the fork has travelled since the last starting pose, and the remaining distance to travel is calculated. The remaining distance to travel is then used to generate the new goal pose after the sideways correction has been made. The algorithm terminates when a forward motion is completed (no more travel distance to the pallet) without a switch detecting an obstruction. 
The routine WAIT-RANGE-SET sets range-set to true when the sonar has returned a range reading indicating the distance the sensor must travel to complete the command. Once the range has been determined SWITCH-READ is called to return the condition of the proximity switches.

Two tool frame motions are executed during this routine; forward translation in the X direction, and sideways translation in the $\mathrm{Z}$ direction. If a switch is closed (indicating proximity to an obstacle) then UPDATE-DISTANCE-TO-GOAL is called (only once according to step-state) to determine the distance the fork has travelled and how much distance remains. FORK-ALIGNMENT then handles the sideways motion until the switch opens indicating the obstacle has been cleared. With no obstacles the fork can now be moved forward towards the new goal by calling MOVE-TO-PALLET, but first the user specified offset in the Z direction is executed by Z-CORRECTION (again the process flow is handled by step-state).

\subsubsection{PICKUP- PALLET-INIT}

\section{Input: PICKUP.PALLET-FILE}

Output: pickup-pallet-para, axis, TRAJ-PHRASE, halt-enable, halt-request, sonar-request, first-time, range-set, z-correcting, step-state, distance-traveled, distance-to-goal

This routine retrieves the parameters compiled in the Path. It uses these parameters to turn on the needed sonars and to retrieve the trajectory parameters.

\subsubsection{PICKUP-PALLET-SONAR-MODEL-INIT \\ Input: snr-model-array \\ Output: snr-model-para}

This routine retrieves the sonar model for the chosen pickup-pallet sonar.

\subsubsection{WAIT-RANGE-SET}

Input: pickup-pallet-para, sonar-valid, min-limit, sonar-range

Output: distance-to-goal, range-set

This routine initializes the value of distance-to-goal using a parameter selected sonar sensor. The sonar-valid flag indicates when valid range data is available. If the flag min-limit equals true then the selected sonar will not work (either because it points in the wrong direction or it is operating outside it's intended range). The parameter p-p-sonar-offset is added to the sonar range so that the fork can be offset from the range calculated goal. range-set is set to true when the distance has been determined.

\subsubsection{MOVE-TO-PALLET}

Input: first-time, tool-pose-^, distance-to-goal, prim-status

Output: TRANSLATE, PRIM-TRAJ, SEND-HALT

This routine commands the robot to move the fork towards the pallet. The goal is calculated as the current tool frame translated a distance equal to distance-to-goal in the plus X direction. PRIM-TRAJ is called to command the robot to move the fork. If prim-status reports done then the goal has been reached and SEND-HALT is called to stop the robot. Note that the process flow will not reach this routine whenever a switch detects an obstacle during the move to the goal.

\subsubsection{Z-CORRECTION}

Input: first-time, delta, p-p-z-correction

Output: TRANSLATE, PRIM-TRAJ, SEND-HALT, first-time, z-correcting 
This routine commands the robot to move the fork in a sideways manner after the proximity switches have cleared an obstacle. The goal is calculated using the parameter p-p-z-correction as the offset to be traveled. The direction is determined from the sign of delta. TRANSLATE is called to generate the goal pose and PRIM-TRAJ is called to command the robot to move the fork. prim-status reports done when the goal has been reached.

\subsubsection{UPDATE-DISTANCE-TO-GOAL}

Input: tool-pose-^, $t$-pose, distance-to-goal

Output: distance-traveled, distance-to-goal

This routine calculates the remaining distance to the goal. The current tool pose is compared to a saved tool pose stored in t-pose. The distance between the poses is calculated and stored in distance-traveled. That value is subtracted from the old distance-to-goal to formulate the new distance-to-goal.

\subsubsection{FORK-ALIGNMENT}

Input: switches, l-tine-mask, r-tine-mask, l-tine-offset Output: delta, SWITCH-CORRECTION

This routine is called when a proximity switch detects an obstacle. The switch input is masked to decouple the left and right tine proximity data. Because of the geometry of the switches and the pallet, both tines cannot simultaneously detect a pallet. If this situation arises the sw-bad error is reported in status-arg (check if there is dirt covering the detector or examine the appropriate LED in the Proximity Interface Box to determine if the sensor is working).

Two tine calibration tables (l-tine-calib-table and r-tine-calib-table) are used to model the $155 \mathrm{~mm}$ pallet foot (see section 2.2. Proximity Detector Modelling). The tables are calibrated to deliver the proper motion to clear the pallet feet dependent on which switches are active. The tables are organized such that the switch data can be used as the index into the table. There are several impossible switch active combinations that are flagged in the tables using a $0 \#$ in the elements. As an example if the current left tine combination equaled 1001, (the outside switches are active), element 17 (1001) would have a 0\# indicating this is impossible. But if the tine combination equaled 0011 (the two right side switches are active) then element 3 (0011) would have a value indicating a translation direction and distance ( -0.75 for example) needed to clear the switches. Thus the routine is divided to test the individual tine switches. The appropriate table signals an error or provides the motion value. An additional offset is provided to allow correction of all the calibration table values. r-tine-offset and l-tine-offset serve this function. They are initialized in the power-up block. SWITCH-CORRECTION is called to command the robot to move the fork and thereby clear the obstacle.

\subsubsection{SWITCH-CORRECTION}

Input: switches, prev-switches, delta Output: TRANSLATE, PRIM-TRAJ, prev-delta, z-correcting, first-time

This routine commands the robot to move the fork to clear a proximity detected obstacle. The variables switches and prev-switches ensure that the same move isn't commanded every cycle, only with the occurrence of a new combination of switches. delta provides the translation direction and distance. Since this routine is only called when the switches are active, if the goal is reached (when prim-status reports done) an error of point-reached is returned. Typically this means that the table value given for clearing the obstacle (dependent on the combination stored in switches) is incorrect. The value or the offset value (r-tine-offset and l-tine-offset) can be made larger but care must be taken not to drive the fork too far such that the opposite tine proximity sensors detect the opposite pallet foot. If this happens the robot can 
become unstable, oscillating between both pallet feet.

\subsubsection{SWITCH-READ}

Input: switches

Output: switches, prev-switches

This routine reads in the physical state of the proximity switches. The value of the old switch readings is saved in the variable prev-switches. The low byte and the high byte is read in and stored into switches. The value is complemented since the port uses an inverter.

5.7.13. The following routines are associated with the GOTO-UNTIL-SW Path Point command (section 3.8.).

\subsubsection{GOTO-UNTIL-SW}

Input: new-ppt, switches, desired-sw-status

Output: EDGE routines

This is the high level routine called during the execution of the GOTO-UNTIL-SW Path Point command. The first time executed it initializes all variables. During all remaining executions it monitors the desired-sw until it matches the desired-sw-status. If the PRIM level reports that the robot has reached the goal pose and the desired switch condition hasn't occurred then an error of no-pallet is reported in status-arg. If the desired switch condition occurs then SEND-HALT is called.

\subsubsection{GOTO-UNTIL-SW-INIT}

Input: GOTO-UNTIL-SW-FILE

Output: sw-para, TRAJ-PHRASE, LOC-PHRASE, PRIM-TRAJ, halt-enable

This routine retrieves the parameters compiled in the Path. It uses these parameters to retrieve the trajectory and location parameters. Finally PRIM-TRAJ is called to command the robot to move to the goal pose.

\subsubsection{RETURN-POSE Path Point Routines}

The following routine is associated with the RETURN-POSE Path Point command (section 3.9.).

\subsubsection{RETURN-POSE}

Input: RETURN-POSE-FILE

Output: ppt-done

This is the high level routine called during the execution of the RETURN-POSE Path Point command. It retrieves the parameter compiled in the Path, return-pose-para- ${ }^{\wedge}$, which is a pointer to a pose record. The current tool frame is then written into the pose record.

\subsubsection{DELAY Path Point Routines}

The following routines are associated with the DELAY Path Point command (section 3.10.).

\subsubsection{DELAY}

Input: delay-\#-cycles

Output: delay-ctr, ppt-done

This is the high level routine called during the execution of the DELAY Path Point command. The first time executed it initializes all variables. During the remaining executions it increments 
delay-ctr until the variable equals delay-\#-cycles. This introduces a delay between the execution of path point commands.

\subsubsection{DELAY-INIT}

Input: DELAY-FILE

Output: delay-para, delay-ctr

This routine retrieves the parameter compiled in the Path.

\subsection{PATH Postprocessing}

FMR conforms to this section.

\subsection{Display and Debug Routines}

This section does not appear in the RCS User's Manual. The purpose of the section is to present routines that are helpful in diagnosing problems with the NBS Sensor Package.

\subsubsection{DISPLAY-SONAR-SELECTION}

\section{Input: sonar-valid}

Output: sonar-range

This routine displays all sonars range values that have been requested (ie. the sonar-request array element for a sonar has been set to true). It should not be compiled into the background control loop for two reasons: First, PRINT does not work in a background task and second, the routine polls on sonar-valid and will stall the control loop until the sonar echo arrives. This routine is primarily used for user verification of working sonars and can be executed directly from the terminal.

\subsubsection{DISPLAY-PROXIMITY-SWITCHES}

Input:

Output: switches

This routine calls SWITCH-READ to update the status of the proximity switches. The value is printed if there is a change in the status of the switches. The routine runs in a loop until it is aborted from the RSL level by typing ABT PATH. Like DISPLAY-SONAR-SELECTION (section 5.9.1.), this routine should not be compiled into the background task. 



\section{RANGE Command}

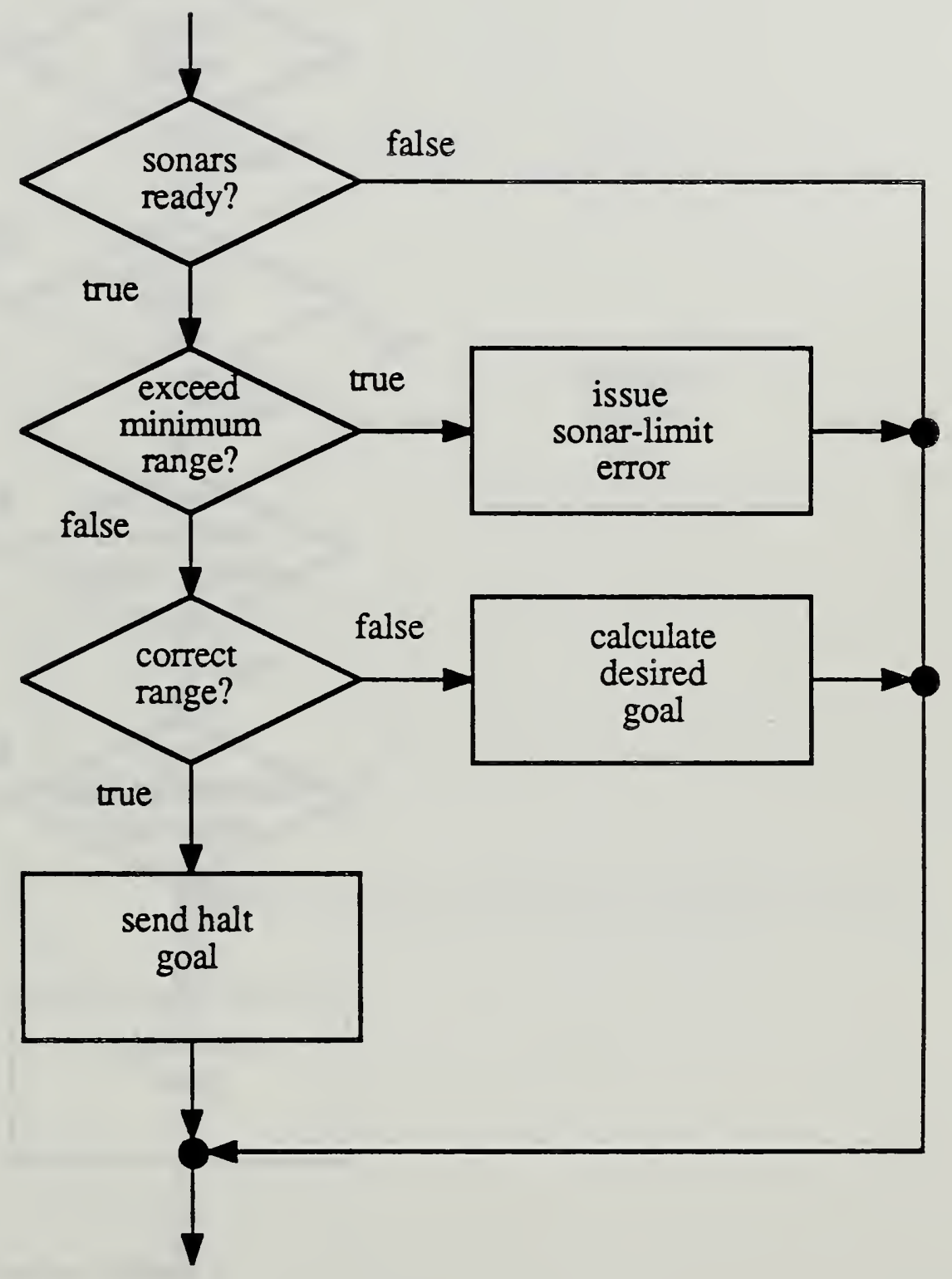




\section{EDGE Command}

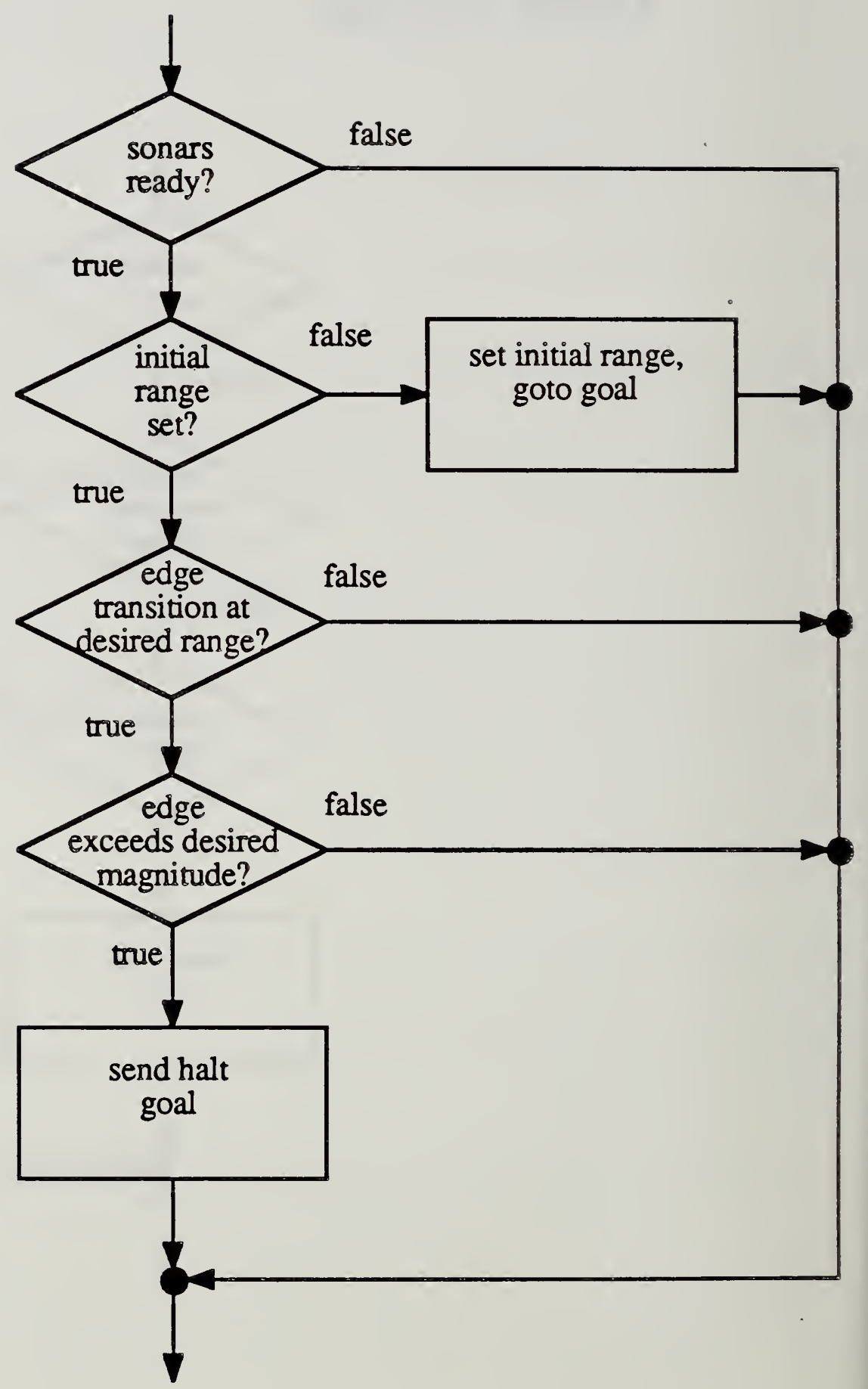




\section{EQUATE Command}

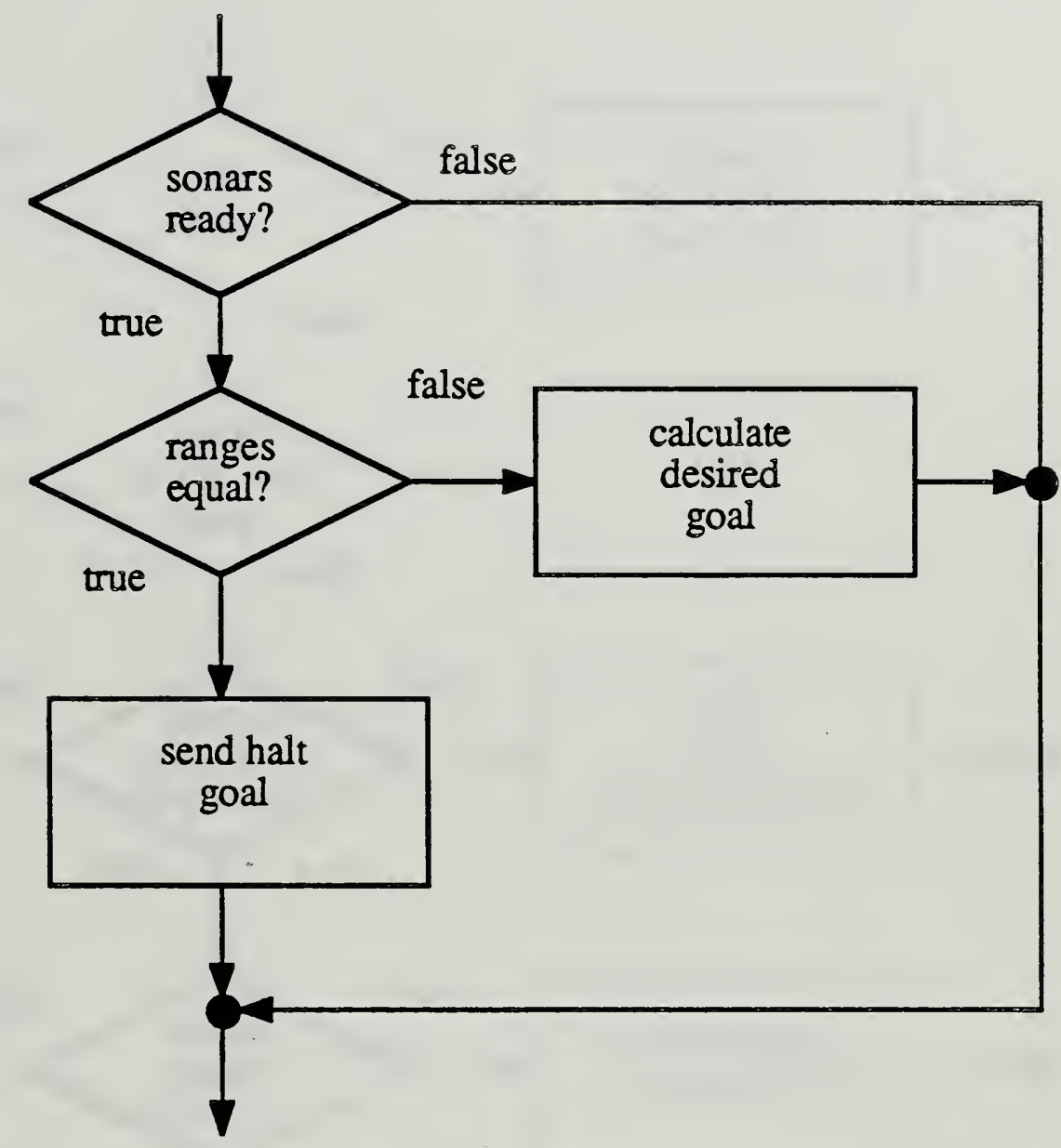


SCAN Command

SCAN-SONAR Routine

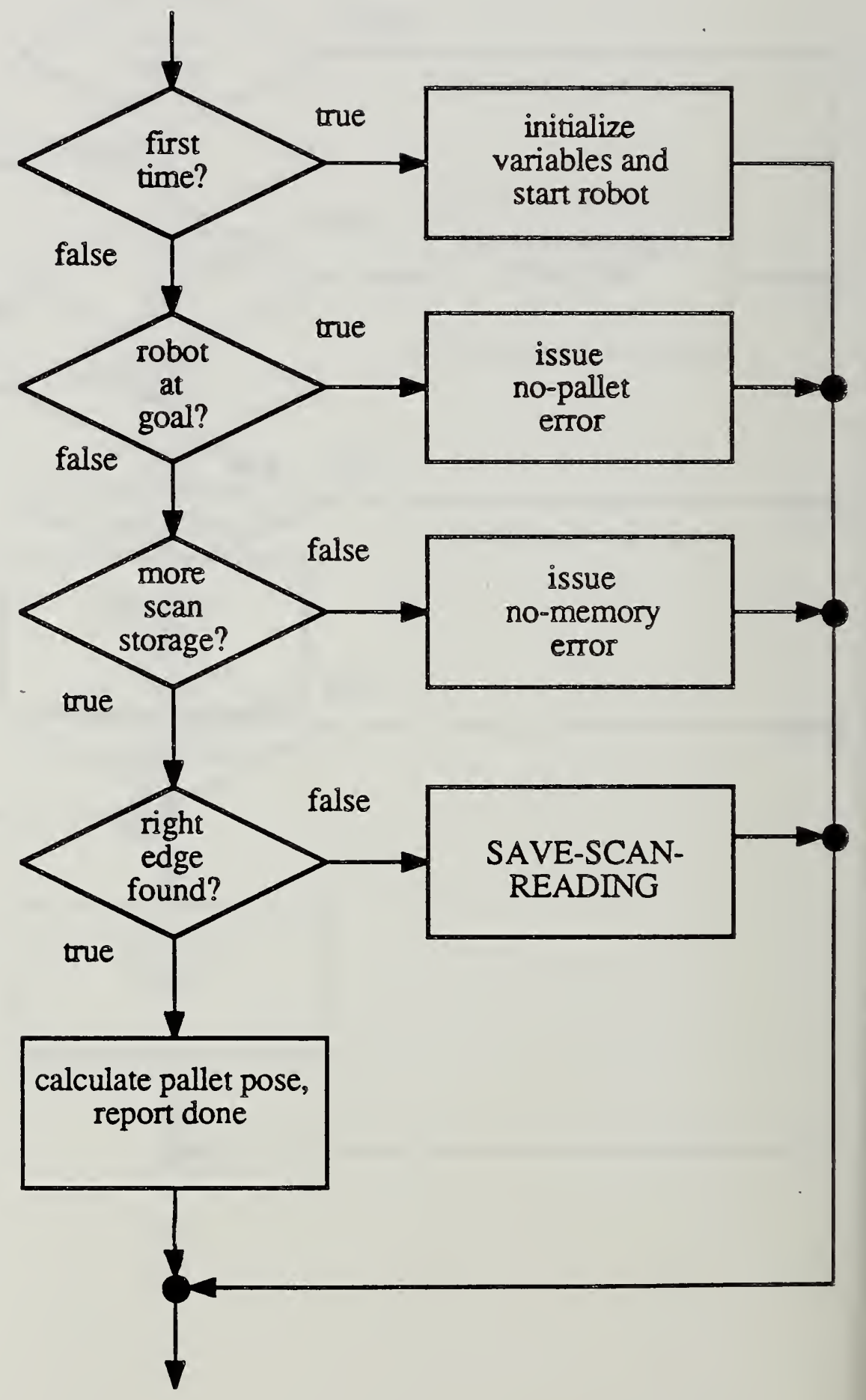




\section{ALIGN-GRIP Command}

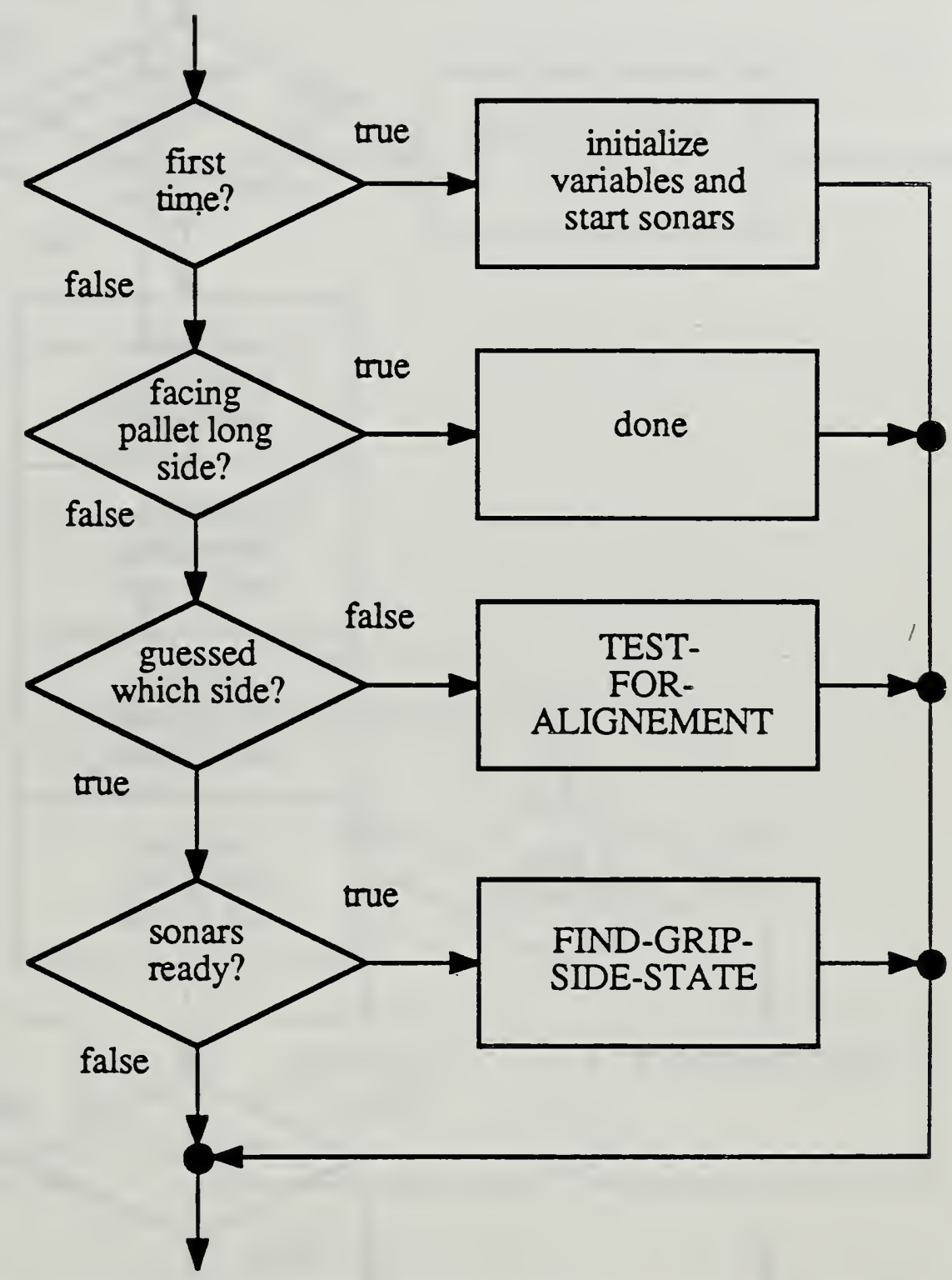




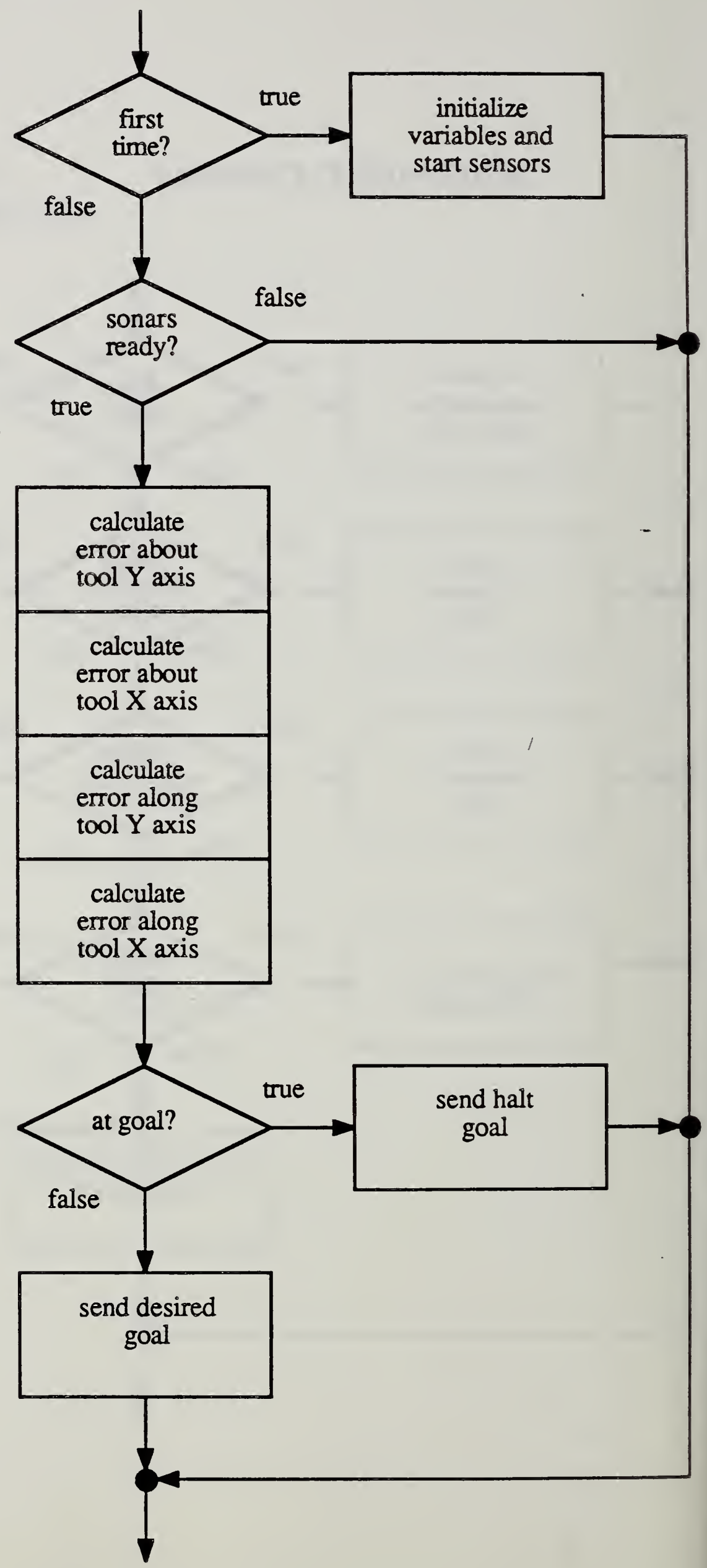




\section{PICKUP-PALLET Command}

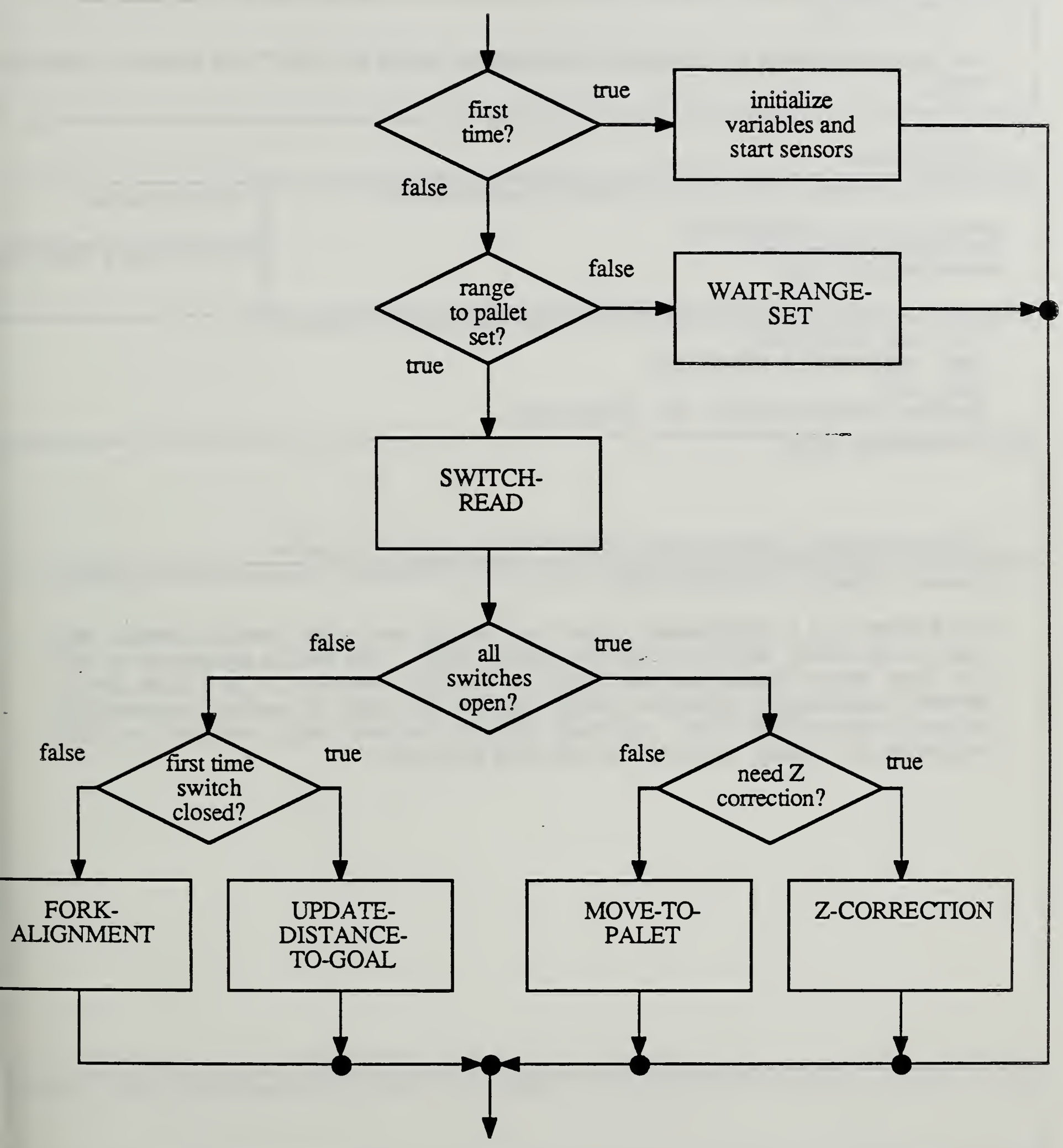


NBS-114A (REV. 2-8C)

U.S. DEPT. OF COMM.

BIBLIOGRAPHIC DATA

SHEET (See instructions)

1. PUBLICATION OR
REPORT NO.
NBSIR $\quad 87-3624$

2. Performing Organ。 Report Nof 3. Publication Date

OCTOBER 1987

4. TITLE AND SUBTITLE

The National Bureau of Standards Programmers Guide for the Field Material Handling Robot.

5. AUTHOR(S)

Sandor Szabo

6. PERFORMING ORGANIZATION (If joint or other than NBS, see instructions)

NATIONAL BUREAU OF STANDARDS

DEPARTMENT OF COMMERCE

WASHINGTON, D.C. 20234

9. SPONSORING ORGANIZATION NAME AND COMPLETE ADDRESS (Street, City, Stote, ZIP)

7. Contracel Grant No.

U.S. Army Laboratory

Human Engineering Laboratory

B.?dg. 459

Aberdeen Proving Ground, MD 21005-5001

10. SUPPLEMENTARY NOTES

Document describes a computer program; SF-185, FIPS Software Summary, is attached.

11. ABSTRACT (A 200.word or less factual summary of most significant information. If document includes a significant bibliogrophy or literoture survey, mention it here)

The document is a programmers guide for the NBS Rea.l-Time Control System (RCS) used in the Field Material Handling Robot (FMR). The FMR is sponsored by the U.S. Army Human Encineering Iaboratory. The RCS (version 2) is a high level, sensory interactive controller which enables the robnt to perform automaticly as a stationary fork lift. The Robot Sensor Iannuare (RSL) provides the RCS a mechanism for sensor integration and task planning.

12. KEY WORDS (Six to twelve entries; alphabetical order; capitalize only proper names; and separate key words ty semicolons)

13. AVAILABILITY

Unlimited

For Official Distribution. Do Nor Release to NTIS

$\square$ Order From Superintendent of Documents, U.S. Government Printing Office, Washington, D.C. 20402.

14. NO. OF

PRINTED PAGES

58

15. Price

$\$ 13.95$

X. Order From National Technical Information Service (NTIS), Springfield, VA. 2216I 

